\title{
Pacific
}

Journal of

Mathematics

\section{GROWTH TIGHT ACTIONS}

Goulnara N. Arzhantseva, Christopher H. CAShen AND Jing TaO 


\section{GROWTH TIGHT ACTIONS}

\section{Goulnara N. Arzhantseva, Christopher H. Cashen And Jing TaO}

We introduce and systematically study the concept of a growth tight action. This generalizes growth tightness for word metrics as initiated by Grigorchuk and de la Harpe. Given a finitely generated, nonelementary group $G$ acting on a $G$-space $\mathcal{X}$, we prove that if $G$ contains a strongly contracting element and if $G$ is not too badly distorted in $\mathcal{X}$, then the action of $G$ on $\mathcal{X}$ is a growth tight action. It follows that if $\mathcal{X}$ is a cocompact, relatively hyperbolic $G$-space, then the action of $G$ on $\mathcal{X}$ is a growth tight action. This generalizes all previously known results for growth tightness of cocompact actions: every already known example of a group that admits a growth tight action and has some infinite, infinite index normal subgroups is relatively hyperbolic; conversely, relatively hyperbolic groups admit growth tight actions. This also allows us to prove that many $\mathrm{CAT}(0)$ groups, including flip-graph manifold groups and many right angled Artin groups, and snowflake groups admit cocompact, growth tight actions. These provide first examples of non relatively hyperbolic groups admitting interesting growth tight actions. Our main result applies as well to cusp uniform actions on hyperbolic spaces and to the action of the mapping class group on Teichmüller space with the Teichmüller metric. Towards the proof of our main result, we give equivalent characterizations of strongly contracting elements and produce new examples of group actions with strongly contracting elements.

0. Introduction

Part I. Growth tight actions

1. Preliminaries

2. Contraction and constriction

3. Abundance of strongly contracting elements

4. A minimal section

5. Embedding a free product set

6. The growth gap

7. The growth of conjugacy classes

MSC2010: 20E06, 20F65, 20F67, 37C35, 57MXX.

Keywords: growth tight, Hopf property, entropy, relatively hyperbolic groups, mapping class groups, snowflake groups, rank 1 isometries, CAT(0) groups. 
Part II. Examples with strongly contracting elements 31

8. Actions on relatively hyperbolic spaces 31

9. Rank 1 actions on CAT(0) spaces 36

10. Mapping class groups 38

11. Snowflake groups 40

Acknowledgments $\quad 45$

References $\quad 45$

\section{Introduction}

The growth exponent of a set $\mathcal{A} \subset \mathcal{X}$ with respect to a pseudometric $d$ is

$$
\delta_{\mathcal{A}, d}:=\limsup _{r \rightarrow \infty} \frac{\log \#\{a \in \mathcal{A} \mid d(o, a) \leqslant r\}}{r}
$$

where \# denotes cardinality and $o \in \mathcal{X}$ is some basepoint. The limit is independent of the choice of basepoint.

Let $G$ be a finitely generated group. A left invariant pseudometric $d$ on $G$ induces a left invariant pseudometric $\bar{d}$ on any quotient $G / \Gamma$ of $G$ by the formula $\bar{d}\left(g \Gamma, g^{\prime} \Gamma\right):=d\left(g \Gamma, g^{\prime} \Gamma\right)$.

Definition 0.1. $G$ is growth tight with respect to $d$ if $\delta_{G, d}>\delta_{G / \Gamma, \bar{d}}$ for every infinite normal subgroup $\Gamma \Vdash G$.

One natural way to put a left invariant metric on a finitely generated group is to choose a finite generating set and consider the word metric. More generally, pseudometrics on a group are provided by actions of the group on metric spaces. Let $\mathcal{X}$ be a $G$-space, that is, a proper, geodesic metric space with a properly discontinuous, isometric $G$-action $G \curvearrowright \mathcal{X}$. The choice of a basepoint $o \in \mathcal{X}$ induces a left invariant pseudometric on $G$ by $d_{G}\left(g, g^{\prime}\right):=d_{\mathcal{X}}\left(g . o, g^{\prime} . o\right)$.

Define the growth exponent $\delta_{G}$ of $G$ with respect to $\mathcal{X}$ to be the growth exponent of $G$ with respect to an induced pseudometric $d_{G}$; this depends only on the $G$-space $\mathcal{X}$, since a different choice of basepoint in $\mathcal{X}$ defines a pseudometric that differs from $d_{G}$ by an additive constant. Likewise, let $\delta_{G / \Gamma}$ denote the growth exponent of $G / \Gamma$ with respect to a pseudometric on $G / \Gamma$ induced by $d_{\mathcal{X}}$.

Definition 0.2. $G \curvearrowright \mathcal{X}$ is a growth tight action if $\delta_{G}>\delta_{G / \Gamma}$ for every infinite normal subgroup $\Gamma \Vdash G$.

Some groups admit growth tight actions for the simple reason that they lack any infinite, infinite index normal subgroups. For such a group $G$, every action on a $G$-space with positive growth exponent will be growth tight. Exponentially growing simple groups are examples, as are irreducible lattices in higher rank semisimple Lie groups, by the Margulis normal subgroup theorem [1991]. 
Growth tightness ${ }^{1}$ for word metrics was introduced and studied by Grigorchuk and de la Harpe [1997], who showed, for example, that a finite rank free group equipped with the word metric from a free generating set is growth tight. On the other hand, they showed that the product of a free group with itself, generated by free generating sets of the factors, is not growth tight. Together with the normal subgroup theorem, these results suggest that for interesting examples of growth tightness we should examine "rank 1" type behavior. Further evidence for this idea comes from the work of Sambusetti and collaborators, who in [Sambusetti 2002b; 2003; 2004; Dal'Bo et al. 2011] proved growth tightness for the action of the fundamental group of a negatively curved Riemannian manifold on its Riemannian universal cover.

In the study of nonpositively curved, or CAT(0), spaces there is a well established idea that a space may be nonpositively curved but have some specific directions that look negatively curved. More precisely:

Definition 0.3 [Ballmann and Brin 1995]. A hyperbolic isometry of a proper CAT(0) space is rank 1 if it has an axis that does not bound a half-flat.

In Definition 2.17, we introduce the notion for an element of $G$ to be strongly contracting with respect to $G \curvearrowright \mathcal{X}$. In the case that $\mathcal{X}$ is a CAT(0) $G$-space, the strongly contracting elements of $G$ are precisely those that act as rank 1 isometries of $\mathcal{X}$; see Theorem 9.1.

In addition to having a strongly contracting element, we will assume that the orbit of $G$ in $\mathcal{X}$ is not too badly distorted. There are two different ways to make this precise.

We say a $G$-space is $C$-quasiconvex if there exists a $C$-quasiconvex $G$-orbit; see Definitions 1.3 and 1.4. This means that it is possible to travel along geodesics joining points in the orbit of $G$ without leaving a neighborhood of the orbit.

Theorem 6.4. Let $G$ be a finitely generated, nonelementary group. Let $\mathcal{X}$ be a quasiconvex $G$-space. If $G$ contains a strongly contracting element, then $G \curvearrowright \mathcal{X}$ is a growth tight action.

Alternatively, we can assume that the growth rate of the number of orbit points that can be reached by geodesics lying entirely, except near the endpoints, outside a neighborhood of the orbit is strictly smaller than the growth rate of the group:

Theorem 6.3. Let $G$ be a finitely generated, nonelementary group. Let $\mathcal{X}$ be a $G$-space. If $G$ contains a strongly contracting element and there exists a $C \geqslant 0$ such

\footnotetext{
${ }^{1}$ Grigorchuk and de la Harpe define growth tightness in terms of "growth rate", which is just the exponentiation of our growth exponent. The growth exponent definition is analogous to the notion of "volume entropy" familiar in Riemannian geometry, and is more compatible with the Poincare series in Section 1B.
} 
that the $C$-complementary growth exponent of $G$ is strictly less than the growth exponent of $G$, then $G \curvearrowright \mathcal{X}$ is a growth tight action.

See Definition 6.2 for a precise definition of the $C$-complementary growth exponent. The proof of Theorem 6.4 is a special case of the proof of Theorem 6.3. Using Theorem 6.4, we prove:

Theorem 8.6. If $\mathcal{X}$ is a quasiconvex, relatively hyperbolic $G$-space and $G$ does not coarsely fix a peripheral subspace, then $G \curvearrowright \mathcal{X}$ is a growth tight action.

This generalizes all previously known results for growth tightness of cocompact actions: every example already known of a group that admits a growth tight action and has some infinite, infinite index normal subgroups is relatively hyperbolic; conversely, relatively hyperbolic groups admit growth tight actions [Arzhantseva and Lysenok 2002; Sambusetti 2002a; Yang 2013; Sambusetti 2003; Sabourau 2013; Dal'Bo et al. 2011].

We also use Theorem 6.4 to prove growth tightness for actions on non-relatively hyperbolic spaces. For instance, we prove that a group action on a proper $\operatorname{CAT}(0)$ space with a rank 1 isometry is growth tight:

Theorem 9.2. If $G$ is a finitely generated, nonelementary group and $\mathcal{X}$ is a quasiconvex, $C A T(0) G$-space such that $G$ contains an element that acts as a rank 1 isometry on $\mathcal{X}$, then $G \curvearrowright \mathcal{X}$ is a growth tight action.

Two interesting classes of non-relatively hyperbolic groups to which Theorem 9.2 applies are nonelementary right angled Artin groups, which are non-relatively hyperbolic when the defining graph is connected, and flip-graph manifolds. These are the first examples of non-relatively hyperbolic groups that admit nontrivial growth tight actions.

Theorem 9.3. Let $\Theta$ be a finite graph that is not a join and has more than one vertex. The action of the right angled Artin group $G$ defined by $\Theta$ on the universal cover $\mathcal{X}$ of the Salvetti complex associated to $\Theta$ is a growth tight action.

Theorem 9.4. Let $M$ be a flip-graph manifold. Let $G$ and $\mathcal{X}$ be the fundamental group and universal cover, respectively, of $M$. Then the action of $G$ on $\mathcal{X}$ by deck transformations is a growth tight action.

We even exhibit an infinite family of non-relatively hyperbolic, non-CAT(0) groups that admit cocompact, growth tight actions:

Theorem 11.1. The Brady-Bridson snowflake groups $B B(1, r)$ for $r \geqslant 3$ admit cocompact, growth tight actions.

Using Theorem 6.3, we prove growth tightness for interesting nonquasiconvex actions. We generalize a theorem of Dal'bo, Peigné, Picaud, and Sambusetti [Dal'Bo et al. 2011] for Kleinian groups satisfying an additional parabolic gap 
condition - see Definition 8.9 - to cusp-uniform actions on arbitrary hyperbolic spaces satisfying the parabolic gap condition:

Theorem 8.10. Let $G$ be a finitely generated, nonelementary group. Let $G \curvearrowright \mathcal{X}$ be a cusp uniform action on a hyperbolic space. Suppose that $G$ satisfies the parabolic gap condition. Then $G \curvearrowright \mathcal{X}$ is a growth tight action.

Once again, our theorems extend beyond actions on relatively hyperbolic spaces, as we use Theorem 6.3 to prove:

Theorem 10.2. The action of the mapping class group of a hyperbolic surface on its Teichmüller space with the Teichmüller metric is a growth tight action.

Mapping class groups, barring exceptional low complexity cases, are neither relatively hyperbolic nor $\mathrm{CAT}(0)$.

In Part I of this paper we prove our main results, Theorem 6.3 and Theorem 6.4. We show in Proposition 3.1 that if there exists a strongly contracting element for $G \curvearrowright \mathcal{X}$ then every infinite normal subgroup $\Gamma$ contains a strongly contracting element $h$. We prove growth tightness by bounding the growth exponent of a subset that is orthogonal, in a coarse sense, to every translate of an axis for $h$.

A dual problem, which is of independent interest, is to find the growth exponent of the conjugacy class of $h$. In Section 7 we show that the growth exponent of the conjugacy class of a strongly contracting element is exactly half the growth exponent of the group, provided the strongly contracting element moves the base point far enough.

In Part II we produce new examples of group actions with strongly contracting elements. These include groups acting on relatively hyperbolic metric spaces (Section 8), certain CAT(0) groups (Section 9), mapping class groups (Section 10), and snowflake groups (Section 11). Our main theorems imply that all these groups admit growth tight actions. These are first examples of growth tight actions and groups which do not come from and are not relatively hyperbolic groups.

0A. Invariance. Growth tightness is a delicate condition. A construction of Dal'bo, Otal, and Peigné [Dal'bo et al. 2000] - see Observation 8.8 - shows that there exist groups $G$ and noncocompact, hyperbolic, equivariantly quasi-isometric $G$-spaces $\mathcal{X}$ and $\mathcal{X}^{\prime}$ such that $G \curvearrowright \mathcal{X}$ is growth tight and $G \curvearrowright \mathcal{X}^{\prime}$ is not.

In [Cashen and Tao 2014], we extend the techniques of this paper to produce the first examples of groups that admit a growth tight action on one of their Cayley graphs and a non-growth tight action on another. This answers in the affirmative the following question of Grigorchuk and de la Harpe [1997]:

Question 1. Does there exist a word metric for which $F_{2} \times F_{2}$ is growth tight? 
Recall that $F_{2} \times F_{2}$ is not growth tight with respect to a generating set that is a union of free generating sets of the two factors.

More generally, a product of infinite groups acting on the $l^{1}$ product of their Cayley graphs is not growth tight. Such $l^{1}$ products and the Dal'bo, Otal, Peigné examples are the only known general constructions of non-growth tight examples. It would be interesting to have a condition to exclude growth tightness. One can not hope to bound the growth exponents of quotients away from that of the group, as Shukhov [1999] and Coulon [2013] have given examples of hyperbolic groups and sequences of quotients whose growth exponents limit to that of the group. At present, growth tightness can only be excluded for a particular action by exhibiting a quotient of the group by an infinite normal subgroup whose growth exponent is equal to that of the group.

0B. The Hopf property. A group $G$ is Hopfian if there is no proper quotient of $G$ isomorphic to $G$.

Let $\mathfrak{D}$ be a set of pseudometrics on $G$ that is quotient-closed, in the sense that if $\Gamma$ is a normal subgroup of $G$ such that there exists an isomorphism $\phi: G \rightarrow G / \Gamma$, then for every $d \in \mathfrak{D}$, the pseudometric on $G$ obtained by pulling back via $\phi$ the pseudometric on $G / \Gamma$ induced by $d$ is also in $\mathfrak{D}$. For example, the set of word metrics on $G$ coming from finite generating sets is quotient-closed.

Suppose further that $\mathfrak{D}$ contains a minimal growth pseudometric $d_{0}$, i.e., that $\delta_{G, d_{0}}=\inf _{d \in \mathfrak{D}} \delta_{G, d}$, and that $G$ is growth tight with respect to $d_{0}$.

Proposition 0.4. Let $G$ be a finitely generated group with a bound on the cardinalities of its finite normal subgroups. Suppose that there exists a quotient-closed set $\mathfrak{D}$ of pseudometrics on $G$ that contains a growth tight, minimal growth element $d_{0}$, as above. Then, $G$ is Hopfian.

The hypothesis on bounded cardinalities of finite normal subgroups holds for all groups of interest in this paper; see Theorem 1.12.

Proof. Suppose that $\Gamma$ is a normal subgroup of $G$ such that $G \cong G / \Gamma$. Let $d$ be the pseudometric on $G$ obtained from pulling back the pseudometric on $G / \Gamma$ induced by $d_{0}$. Since $\mathfrak{D}$ is quotient-closed, $d \in \mathfrak{D}$. By minimality, $\delta_{G, d_{0}} \leqslant \delta_{G, d}$, but by growth tightness, $\delta_{G, d} \leqslant \delta_{G, d_{0}}$, with equality only if $\Gamma$ is finite. Thus, the only normal subgroups $\Gamma$ for which we could have $G \cong G / \Gamma$ are finite. However, if $G \cong G / \Gamma$ for some finite $\Gamma$ then $G$ has arbitrarily large finite normal subgroups, contrary to hypothesis.

Grigorchuk and de la Harpe [1997] suggested this as a possible approach to the question of whether a nonelementary Gromov hyperbolic group is Hopfian, in the particular case that $\mathfrak{D}$ is the set of word metrics on $G$. Arzhantseva and Lysenok [2002] proved that every word metric on a nonelementary hyperbolic 
group is growth tight. They conjectured that the growth exponent of such a group achieves its infimum on some finite generating set and proved a step towards this conjecture [Arzhantseva and Lysenok 2006]. Sambusetti [2002a] gave an examples of a (nonhyperbolic) group for which the set of word metrics does not realize its infimal growth exponent. In general, it is difficult to determine whether a given group has a generating set that realizes the infimal growth exponent among word metrics. Part of our motivation for studying growth tight actions is to open new possibilities for the set $\mathfrak{D}$ of pseudometrics considered above.

Torsion free hyperbolic groups are Hopfian [Sela 1999]. Reinfeldt and Weidmann [2014] have announced a generalization of Sela's techniques to hyperbolic groups with torsion, and concluded that all hyperbolic groups are Hopfian.

0C. The rank rigidity conjecture. The rank rigidity conjecture (see [Caprace and Sageev 2011; Ballmann and Buyalo 2008]) asserts that if $\mathcal{X}$ is a locally compact, irreducible, geodesically complete $\mathrm{CAT}(0)$ space, and $G$ is an infinite discrete group acting properly and cocompactly on $\mathcal{X}$, then one of the following holds:

(1) $\mathcal{X}$ is a higher rank symmetric space.

(2) $\mathcal{X}$ is a Euclidean building of dimension at least 2.

(3) $G$ contains a rank 1 isometry.

In case (1), the Margulis normal subgroup theorem implies that $G$ is trivially growth tight, since it has no infinite, infinite index normal subgroups. Conjecturally, the Margulis normal subgroup theorem also holds in case (2). Our Theorem 9.2 says that if $\mathcal{X}$ is proper then $G \curvearrowright \mathcal{X}$ is a growth tight action in case (3). Thus, a non-growth tight action of a nonelementary group on a proper, irreducible CAT $(0)$ space as above would provide a counterexample either to the rank rigidity conjecture or to the conjecture that the Margulis normal subgroup theorem applies to Euclidean buildings.

The rank rigidity conjecture is known to be true for many interesting classes of spaces, such as Hadamard manifolds [Ballmann 1995], 2-dimensional, piecewise Euclidean cell complexes [Ballmann and Brin 1995], Davis complexes of Coxeter groups [Caprace and Fujiwara 2010], universal covers of Salvetti complexes of right angled Artin groups [Behrstock and Charney 2012], and finite dimensional CAT(0) cube complexes [Caprace and Sageev 2011]; so Theorem 9.2 provides many new examples of growth tight actions.

It is unclear when growth tightness holds if $\mathcal{X}$ is reducible. A direct product of infinite groups acting via a product action on a product space with the $l^{1}$ metric fails to be growth tight. However, there are also examples [Burger and Mozes 1997] of infinite simple groups acting cocompactly on products of trees. In [Cashen and Tao 2014], we find partial results in the case that the group action is a product action. 
0D. Outline of the proof of the main theorems. Sambusetti [2002a] proved that a nonelementary free product of nontrivial groups has a greater growth exponent than that of either factor. Thus, a strategy to prove growth tightness is to find a subset of $G$ that looks like a free product, with one factor that grows like the quotient group we are interested in. Specifically:

(1) Find a subset $A \subset G \subset \mathcal{X}$ such that $\delta_{A}=\delta_{G / \Gamma}$. We will obtain $A$ as a coarsely dense subset of a minimal section of the quotient map $G \rightarrow G / \Gamma$; see Definition 4.4.

(2) Construct an embedding of a free product set $A * \mathbb{Z}_{2}$ into $\mathcal{X}$. The existence of a strongly contracting element $h \in \Gamma$ is used in the construction of this embedding; see Proposition 5.1.

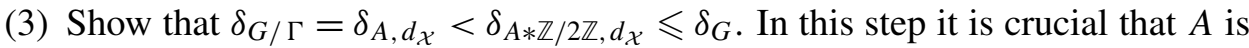
divergent; see Definition 1.7 and Lemma 6.1. We use the quasiconvexity and complementary growth exponent to establish divergence.

This outline, due to Sambusetti, is nowadays standard. Typically, step (2) is accomplished by a ping-pong argument, making use of fine control on the geometry of the space $\mathcal{X}$. Our methods are coarser than such a standard approach, and therefore can be applied to a wider variety of spaces. We use, in particular, a technique of Bestvina, Bromberg, and Fujiwara [Bestvina et al. 2014] to construct an action of $G$ on a quasitree. Verifying that the map from the free product set into $\mathcal{X}$ is an embedding amounts to showing that elements in $A$ do not cross certain coarse edges of the quasitree.

\section{Part I. Growth tight actions}

\section{Preliminaries}

Fix a $G$-space $\mathcal{X}$. From now on, $d$ is used to denote the metric on $\mathcal{X}$ as well as the induced pseudometric on $G$ and $G / \Gamma$. Since there will be no possibility of confusion, we suppress $d$ from the growth exponent notation.

We denote by $\mathcal{B}_{r}(x)$ the open ball of radius $r$ about the point $x$ and by $\mathcal{B}_{r}(\mathcal{A}):=$ $\bigcup_{x \in \mathcal{A}} \mathcal{B}_{r}(x)$ the open $r$-neighborhood about the set $\mathcal{A}$. The closed $r$-ball and closed $r$-neighborhood are denoted $\overline{\mathcal{B}}_{r}(x)$ and $\overline{\mathcal{B}}_{r}(\mathcal{A})$, respectively.

1A. Coarse language. All of the following definitions may be written without specifying $C$ to indicate that some such $C \geqslant 0$ exists: Two subsets $\mathcal{A}$ and $\mathcal{A}^{\prime}$ of $\mathcal{X}$ are $C$-coarsely equivalent if $\mathcal{A} \subset \overline{\mathcal{B}}_{C}\left(\mathcal{A}^{\prime}\right)$ and $\mathcal{A}^{\prime} \subset \overline{\mathcal{B}}_{C}(\mathcal{A})$. A subset $\mathcal{A}$ of $\mathcal{X}$ is $C$-coarsely dense if it is $C$-coarsely equivalent to $\mathcal{X}$. A subset $\mathcal{A}$ of $\mathcal{X}$ is $C$-coarsely connected if for every $a$ and $a^{\prime}$ in $\mathcal{A}$ there exists a chain $a=a_{0}, a_{1}, \ldots, a_{n}=a^{\prime}$ of points in $\mathcal{A}$ with $d\left(a_{i}, a_{i+1}\right) \leqslant C$. 
A pseudomap $\phi: \mathcal{X} \rightarrow \mathcal{Y}$ assigns to each point in $\mathcal{X}$ a subset $\phi(x)$ of $\mathcal{Y}$. A pseudomap is $C$-coarsely well-defined if for every $x \in \mathcal{X}$ the set $\phi(x)$ of $\mathcal{Y}$ has diameter at most $C$. Pseudomaps $\phi$ and $\phi^{\prime}$ with the same domain and codomain are $C$-coarsely equivalent or $C$-coarsely agree if $\phi(x)$ is $C$-coarsely equivalent to $\phi^{\prime}(x)$ for every $x$ in the domain. A $C$-coarsely well-defined pseudomap is called a $C$-coarse map. From a $C$-coarse map we can obtain a $C$-coarsely equivalent map by selecting one point from every image set. Conversely:

Lemma 1.1. If $\phi: \mathcal{X} \rightarrow \mathcal{Y}$ is coarsely $G$-equivariant then there is an equivariant coarse map coarsely equivalent to $\phi$.

Proof. Suppose there is a $C$ such that $d(g . \phi(x), \phi(g . x)) \leqslant C$ for all $x \in \mathcal{X}$ and $g \in G$. Define $\phi^{\prime}(x):=\bigcup_{g \in G} g^{-1} . \phi(g \cdot x)$. Then, $\phi^{\prime}$ is $G$-equivariant and $C$-coarsely equivalent to $\phi$.

Notation 1.2. If $\phi: \mathcal{X} \rightarrow \mathcal{Y}$ is a pseudomap and $\mathcal{A}$ and $\mathcal{A}^{\prime}$ are subsets of $\mathcal{X}$, let $d^{\phi}\left(\mathcal{A}, \mathcal{A}^{\prime}\right)$ denote the diameter of $\phi(\mathcal{A}) \cup \phi\left(\mathcal{A}^{\prime}\right)$.

Definition 1.3. A subset $\mathcal{A} \subset \mathcal{X}$ is $C$-quasiconvex if for every $a_{0}, a_{1} \in \mathcal{A}$, there exists a geodesic $\gamma$ between $a_{0}$ and $a_{1}$ such that $\gamma \subset \overline{\mathcal{B}}_{C}(\mathcal{A})$. It is $C$-strongly quasiconvex if every geodesic with endpoints in $\mathcal{A}$ stays in $\overline{\mathcal{B}}_{C}(\mathcal{A})$.

Definition 1.4. A $G$-space $\mathcal{X}$ is $C$-quasiconvex if it contains a $C$-quasiconvex $G$-orbit.

For convenience, if $\mathcal{X}$ is a quasiconvex $G$-space we assume we have chosen a basepoint $o \in \mathcal{X}$ such that $G . o$ is quasiconvex.

A group is elementary if it has a finite index cyclic subgroup.

Definition 1.5. Let $g \in G$. The elementary closure of $g$, denoted by $E(g)$, is the largest virtually cyclic subgroup containing $g$, if such a subgroup exists.

A map $\phi: \mathcal{X} \rightarrow \mathcal{Y}$ is an $(M, C)$-quasi-isometric embedding, for some $M \geqslant 1$ and $C \geqslant 0$, if, for all $x_{0}, x_{1} \in \mathcal{X}$ :

$$
\frac{1}{M} d\left(x_{0}, x_{1}\right)-C \leqslant d\left(\phi\left(x_{0}\right), \phi\left(x_{1}\right)\right) \leqslant M d\left(x_{0}, x_{1}\right)+C
$$

A map $\phi$ is $C$-coarsely M-Lipschitz if the second inequality holds, and is a quasiisometry if it is a quasi-isometric embedding whose image is $C$-coarsely dense.

An $(M, C)$-quasigeodesic is an $(M, C)$-quasi-isometric embedding of a coarsely connected subset of $\mathbb{R}$. If $\gamma: I \rightarrow \mathcal{X}$ is a quasigeodesic we let $\gamma_{t}$ denote the point $\gamma(t)$, and let $\gamma$ denote the image of $\gamma$ in $\mathcal{X}$.

Definition 1.6. A quasigeodesic $\mathcal{Q}$ is Morse if for every $M \geqslant 1$ there exists a $K \geqslant 0$ such that every $(M, M)$-quasigeodesic with endpoints on $\mathcal{Q}$ is contained in the $K$-neighborhood of $\mathcal{Q}$. 
We will use notation to simplify some calculations. Let $C$ be a "universal constant". For us this will usually mean a constant that depends on $G \curvearrowright \mathcal{X}$ and a choice of $o \in \mathcal{X}$, but not on the point in $\mathcal{X}$ at which quantities $a$ and $b$ are calculated.

- For $a \leqslant C b$, we write $a \stackrel{*}{\prec} b$.

- For $\frac{1}{C} b \leqslant a \leqslant C b$, we write $a \stackrel{*}{`} b$.

- For $a \leqslant b+C$, we write $a \stackrel{\dagger}{\prec}$.

- For $b-C \leqslant a \leqslant b+C$, we write $a \succsim b$.

- For $a \leqslant C b+C$, we write $a \prec b$.

- For $\frac{1}{C} b-C \leqslant a \leqslant C b+C$, we write $a \asymp b$.

1B. The Poincaré series and growth. Let $(\mathcal{X}, o, d)$ be a pseudometric space with choice of basepoint. Let $|x|:=d(o, x)$ be the induced seminorm. Define the Poincaré series of $\mathcal{A} \subset \mathcal{X}$ to be

$$
\Theta_{\mathcal{A}}(s):=\sum_{a \in \mathcal{A}} \exp (-s|a|)
$$

Another related series is:

$$
\Theta_{\mathcal{A}}^{\prime}(s):=\sum_{n=0}^{\infty} \#\left(\overline{\mathcal{B}}_{n}(o) \cap \mathcal{A}\right) \cdot \exp (-s n)
$$

The series $\Theta_{\mathcal{A}}$ and $\Theta_{\mathcal{A}}^{\prime}$ have the same convergence behavior, since $\Theta_{\mathcal{A}}(s)=$ $\Theta_{\mathcal{A}}^{\prime}(s) \cdot(1-\exp (-s))$. It follows that the growth exponent of $\mathcal{A}$ is a critical exponent for $\Theta_{\mathcal{A}}^{\prime}$ and $\Theta_{\mathcal{A}}$ : the series converge for $s$ greater than the critical exponent and diverge for $s$ less than the critical exponent.

Definition 1.7. $\mathcal{A} \subset \mathcal{X}$ is divergent if $\Theta_{\mathcal{A}}$ diverges at its critical exponent.

Since point stabilizers are finite, if $A<G$ and we set $\mathcal{A}:=A$.o then $\Theta_{A} \stackrel{*}{\rightleftharpoons} \Theta_{\mathcal{A}}$ and $\Theta_{A}^{\prime} \stackrel{*}{=} \Theta_{\mathcal{A}}^{\prime}$. This implies $\delta_{A}=\delta_{\mathcal{A}}$, so we can compute the growth exponent of $A$ with respect to the pseudometric on $A$ induced by $G \curvearrowright \mathcal{X}$ by computing the growth exponent of the $A$-orbit as a subset of $\mathcal{X}$.

1C. The quasitree construction. We recall the method of Bestvina, Bromberg, and Fujiwara [Bestvina et al. 2014] for producing group actions on quasitrees. A quasitree is a geodesic metric space that is quasi-isometric to a simplicial tree. Manning [2005] gave a characterization of quasitrees as spaces satisfying a "bottleneck" property. We use an equivalent formulation:

Definition 1.8. A geodesic metric space satisfies the bottleneck property if there exists a number $\Delta$ such that for all $x$ and $y$ in $\mathcal{X}$, and for any point $m$ on a geodesic segment from $x$ to $y$, every path from $x$ to $y$ passes through $\overline{\mathcal{B}}_{\Delta}(m)$. 
Theorem 1.9 [Manning 2005, Theorem 4.6]. A geodesic metric space is a quasitree if and only if it satisfies the bottleneck property.

Let $\mathbb{Y}$ be a collection of geodesic metric spaces, and suppose for each $X, Y \in \mathbb{Y}$ we have a subset $\pi_{Y}(X) \subset Y$, which is referred to as the projection of $X$ to $Y$. Let $d_{Y}^{\pi}(X, Z):=\operatorname{diam} \pi_{Y}(X) \cup \pi_{Y}(Z)$.

Definition 1.10 (projection axioms). A set $\mathbb{Y}$ with projections as above satisfies the projection axioms if there exist $\xi \geqslant 0$ such that for all distinct $X, Y, Z \in \mathbb{Y}$ :

(P0) $\operatorname{diam} \pi_{Y}(X) \leqslant \xi$

(P1) At most one of $d_{X}^{\pi}(Y, Z), d_{Y}^{\pi}(X, Z)$, or $d_{Z}^{\pi}(X, Y)$ is strictly greater than $\xi$.

(P2) $\left|\left\{V \in \mathbb{Y} \mid d_{V}^{\pi}(X, Y)>\xi\right\}\right|<\infty$

For a motivating example, let $G$ be the fundamental group of a closed hyperbolic surface, and let $\mathcal{H}$ be the axis in $\mathbb{\boxplus}^{2}$ of $h \in G$. Let $\mathbb{Y}$ be the distinct $G$-translates of $\mathcal{H}$, and for each $Y \in \mathbb{Y}$, let $\pi_{Y}$ be closest point projection to $Y$. In this example, projection distances arise as closest point projection in an ambient space containing $\mathbb{Y}$. Bestvina, Bromberg, and Fujiwara consider abstractly the collection $\mathbb{Y}$ and projections satisfying the projection axioms, and build an ambient space containing a copy of $\mathbb{Y}$ such that closest point projection agrees with the given projections, up to bounded error:

Theorem 1.11 [Bestvina et al. 2014, Theorems A and B]. Consider a set $\mathbb{Y}$ of geodesic metric spaces and projections satisfying the projection axioms. There exists a geodesic metric space $\mathcal{Y}$ containing disjoint, isometrically embedded, totally geodesic copies of each $Y \in \mathbb{Y}$, such that for $X, Y \in \mathbb{Y}$, closest point projection of $X$ to $Y$ in $\mathcal{Y}$ is uniformly coarsely equivalent to $\pi_{Y}(X)$.

The construction is equivariant with respect to any group action that preserves the projections. Also, if each $Y \in \mathbb{Y}$ is a quasitree, with uniform bottleneck constants, then $\mathcal{Y}$ is a quasitree.

The basic idea is that $Z$ is "between" $X$ and $Y$ in $\mathbb{Y}$ if $d_{Z}^{\pi}(X, Y)$ is large, and $X$ and $Y$ are "close" if there is no $Z$ between them. Essentially, $\mathcal{Y}$ is constructed by choosing parameters $C$ and $K$ and connecting every point of $\pi_{Y}(X)$ to every point of $\pi_{X}(Y)$ by an edge of length $K$ if there does not exist $Z \in \mathbb{Y}$ with $d_{Z}^{\pi}(X, Y)>C$. For technical reasons one actually must perturb the projection distances by a bounded amount first. Then, if $C$ is chosen sufficiently large and $K$ is chosen sufficiently large with respect to $C$, the resulting space is the space $\mathcal{Y}$ of Theorem 1.11.

1D. Hyperbolically embedded subgroups. Dahmani, Guirardel, and Osin [Dahmani et al. 2011] define the concept of a hyperbolically embedded subgroup. This is a generalization of a peripheral subgroup of a relatively hyperbolic group. We will not state the definition, as it is technical and we will not work with this 
property directly, but it follows from Theorem 4.42 from that reference that $E(h)$ is hyperbolically embedded in $G$ for any strongly contracting element $h$. The proof of this theorem proceeds by considering the action of $E(h)$ on a quasitree constructed via the method of Bestvina, Bromberg, and Fujiwara.

We state some results on hyperbolically embedded subgroups that are related to the work in this paper. These are not used in the proofs of the main theorems.

Theorem 1.12 [Dahmani et al. 2011, Theorem 2.23]. If $G$ has a hyperbolically embedded subgroup, then $G$ has a maximal finite normal subgroup.

Recall that this theorem guarantees one of the hypotheses of Proposition 0.4.

Theorem 1.13. If $G$ contains an infinite order element $h$ such that $E(h)$ is hyperbolically embedded, then $G$ has an infinite, infinite index normal subgroup.

Proof. By [Dahmani et al. 2011, Theorem 5.15], for a sufficiently large $n$, the normal closure of $\left\langle h^{n}\right\rangle$ in $G$ is the free product of the conjugates of $\left\langle h^{n}\right\rangle$.

This theorem says that our main results are true for interesting reasons, not simply for lack of normal subgroups.

Minasyan and Osin [2015] produce hyperbolically embedded subgroups in certain graphs of groups. We use these to produce growth tight examples in Theorem 9.5.

Theorem 1.14 [Minasyan and Osin 2015, Theorem 4.17]. Let $G$ be a finitely generated, nonelementary group that splits nontrivially as a graph of groups and is not an ascending $H N N$-extension. If there exist two edges of the corresponding Bass-Serre tree whose stabilizers have finite intersection then $G$ contains an infinite order element $h$ such that $E(h)$ is hyperbolically embedded in $G$.

\section{Contraction and constriction}

In this section we introduce properties called "contracting" and "constricting" that generalize properties of closest point projection to a geodesic in hyperbolic space, and verify that the "strong" versions of these properties are sufficient to satisfy the projection axioms of Definition 1.10. These facts are well known to the experts ${ }^{2}$, but as there is currently no published general treatment of this material, we provide a detailed account.

2A. Contracting and constricting. In this section we define contracting and constricting maps and show that the strong versions of these properties are equivalent.

Definition 2.1. A $C$-coarse map $\pi: \mathcal{X} \rightarrow \mathcal{A}$ is $C$-coarsely a closest point projection if for all $x$ there exists an $a \in \mathcal{A}$ with $d(x, \mathcal{A})=d(x, a)$ such that $\operatorname{diam}\{a\} \cup \pi(x) \leqslant C$.

\footnotetext{
${ }^{2}$ For example, [Sisto 2011] shows that the projection axioms are satisfied for constricting elements, without assuming that $\mathcal{X}$ is proper.
} 
Recall $d^{\pi}\left(x_{0}, x_{1}\right):=\operatorname{diam} \pi\left(x_{0}\right) \cup \pi\left(x_{1}\right)$.

Definition 2.2. $\pi: \mathcal{X} \rightarrow \mathcal{A}$ is $(M, C)$-contracting for $C \geqslant 0$ and $M \geqslant 1$ if

(1) $\pi$ and $\operatorname{Id}_{\mathcal{A}}$ are $C$-coarsely equivalent on $\mathcal{A}$, and

(2) $d\left(x_{0}, x_{1}\right)<\frac{1}{M} d\left(x_{0}, \mathcal{A}\right)-C$ implies $d^{\pi}\left(x_{0}, x_{1}\right) \leqslant C$ for all $x_{0}, x_{1} \in \mathcal{X}$.

We say $\pi$ is strongly contracting if it is $(1, C)$-contracting and, for all $x \in \mathcal{X}$, $d(x, \pi(x))-d(x, \mathcal{A}) \leqslant C$.

Another formulation of strong contraction says that geodesics far from $\mathcal{A}$ have bounded projections to $\mathcal{A}$ :

Definition 2.3. A coarse map $\pi: \mathcal{X} \rightarrow \mathcal{A}$ has the bounded geodesic image property if there is a constant $C$ such that for every geodesic $\mathcal{L}$, if $\mathcal{L} \cap \mathcal{B}_{C}(\mathcal{A})=\varnothing$ then $\operatorname{diam}(\pi(\mathcal{L})) \leqslant C$.

Lemma 2.4. If $d(x, \pi(x))-d(x, \mathcal{A})$ is uniformly bounded, then $\pi$ has the bounded geodesic image property if and only if it is strongly contracting.

Proof. First, assume that $\pi$ has the bounded geodesic image property, for some constant $C$. Let $x$ be any point in $\mathcal{X} \backslash \mathcal{B}_{C}(\mathcal{A})$. For any $y$ such that $d(x, y)<$ $d(x, \mathcal{A})-C$, every geodesic from $x$ to $y$ remains outside $\mathcal{B}_{C}(\mathcal{A})$, so its projection has diameter at most $C$.

For the converse, suppose $\pi: \mathcal{X} \rightarrow \mathcal{A}$ is a $C$-coarse map that is $(1, C)$-contracting and $d(x, \pi(x))-d(x, \mathcal{A}) \leqslant C$ for all $x \in \mathcal{X}$. If $C=0$ then balls outside of $\mathcal{B}_{C}(\mathcal{A})$ project to a single point, and we are done; so assume that $C>0$. Let $\mathcal{L}:[0, T] \rightarrow \mathcal{X}$ be a geodesic that stays outside $\mathcal{B}_{3 C}(\mathcal{A})$. Let $t_{0}:=d\left(\mathcal{L}_{0}, \mathcal{A}\right)-C$, and let $s:=T-d\left(\mathcal{L}_{T}, \mathcal{A}\right)+C$. If $s \leqslant t_{0}$ then $d^{\pi}\left(\mathcal{L}_{0}, \mathcal{L}_{T}\right) \leqslant 2 C$. Otherwise, define $t_{i+1}:=t_{i}+d\left(\mathcal{L}_{t_{i}}, \mathcal{A}\right)-C$, provided $t_{i+1}<s$. Each $t_{i+1}-t_{i} \geqslant 2 C$, so we have a partition of $[0, T]$ into subintervals $\left[0, t_{0}\right],\left[t_{0}, t_{1}\right], \ldots,\left[t_{k-1}, t_{k}\right],\left[t_{k}, s\right],[s, T]$ with $k<\left(s-t_{0}\right) /(2 C)$, and if $[a, b]$ is one of these intervals then $d^{\pi}\left(\mathcal{L}_{a}, \mathcal{L}_{b}\right) \leqslant C$, by strong contraction. Now,

$$
\begin{gathered}
d\left(\mathcal{L}_{0}, \mathcal{L}_{T}\right) \leqslant d\left(\mathcal{L}_{0}, \pi\left(\mathcal{L}_{0}\right)\right)+d\left(\pi\left(\mathcal{L}_{0}\right), \pi\left(\mathcal{L}_{t_{0}}\right)\right)+d\left(\pi\left(\mathcal{L}_{t_{0}}\right), \pi\left(\mathcal{L}_{s}\right)\right) \\
\quad+d\left(\pi\left(\mathcal{L}_{s}\right), \pi\left(\mathcal{L}_{T}\right)\right)+d\left(\pi\left(\mathcal{L}_{T}\right), \mathcal{L}_{T}\right) \\
\leqslant d\left(\mathcal{L}_{0}, \pi\left(\mathcal{L}_{0}\right)\right)+d\left(\pi\left(\mathcal{L}_{T}\right), \mathcal{L}_{T}\right)+C\left(2+\frac{s-t_{0}}{2 C}\right),
\end{gathered}
$$

and

$$
\begin{aligned}
d\left(\mathcal{L}_{0}, \mathcal{L}_{T}\right) & =d\left(\mathcal{L}_{0}, \mathcal{L}_{t_{0}}\right)+d\left(\mathcal{L}_{t_{0}}, \mathcal{L}_{s}\right)+d\left(\mathcal{L}_{s}, \mathcal{L}_{T}\right) \\
& =d\left(\mathcal{L}_{0}, \mathcal{A}\right)-C+s-t_{0}+d\left(\mathcal{L}_{T}, \mathcal{A}\right)-C,
\end{aligned}
$$

so

$$
s-t_{0} \leqslant 2\left(5 C+d\left(\mathcal{L}_{0}, \pi\left(\mathcal{L}_{0}\right)\right)-d\left(\mathcal{L}_{0}, \mathcal{A}\right)+d\left(\mathcal{L}_{T}, \pi\left(\mathcal{L}_{T}\right)\right)-d\left(\mathcal{L}_{T}, \mathcal{A}\right)\right) \leqslant 14 C .
$$

This means that $k<7$, so $d^{\pi}\left(\mathcal{L}_{0}, \mathcal{L}_{T}\right) \leqslant C(3+k)<10 C$. 
If $\pi$ is only $(M, C)$-contracting then a similar argument shows that $d^{\pi}\left(\mathcal{L}_{0}, \mathcal{L}_{T}\right)$ is bounded in terms of $C$ and $\log _{(M+1) /(M-1)}\left(d\left(\mathcal{L}_{0}, \mathcal{A}\right) d\left(\mathcal{L}_{T}, \mathcal{A}\right)\right)$.

We now introduce the notion of a constricting map. Using constricting maps will simplify some of our proofs, but it turns out that the strong versions of constricting and contracting are equivalent.

Definition 2.5. A path system is a transitive collection of quasigeodesics with uniform constants that is closed under taking subpaths.

A path system is minimizing if, for some $C \geqslant 0$, it contains a path system consisting of $(1, C)$-quasigeodesics.

Definition 2.6. Let $\mathcal{P S}$ be a path system. For $M \geqslant 1$ and $C \geqslant 0$, a coarse map $\pi: \mathcal{X} \rightarrow \mathcal{A}$ is $(M, C)$-PS-constricting ${ }^{3}$ if:

(1) $\mathcal{P S}$ contains a path system consisting of $(M, C)$-quasigeodesics,

(2) $\pi$ and $\operatorname{Id}_{\mathcal{A}}$ are $C$-coarsely equivalent on $\mathcal{A}$, and

(3) for every $\mathcal{P} \in \mathcal{P S}$ with endpoints $x_{0}$ and $x_{1}$, if $d^{\pi}\left(x_{0}, x_{1}\right)>C$ then, for both $i \in\{0,1\}$, we have $d\left(\pi\left(x_{i}\right), \mathcal{P}\right) \leqslant C$.

A coarse map is constricting if it is $(M, C)-\mathcal{P S}$-constricting for some path system $\mathcal{P S}$ and strongly constricting if it is $(1, C)$-constricting for the path system consisting of all geodesics.

Lemma 2.7. If $\pi: \mathcal{X} \rightarrow \mathcal{A}$ is constricting, then it is contracting.

Proof. Suppose $\pi$ is $(M, C)-\mathcal{P S}$-constricting $C$-coarse map for a path system $\mathcal{P S}$ consisting of $(M, C)$-quasigeodesics. Suppose $\mathcal{P}:[0, T] \rightarrow \mathcal{X}$ is a path in $\mathcal{P S}$ with $\mathcal{P}_{0}=x$ and $\mathcal{P}_{T}=y$, and suppose $z=\mathcal{P}_{s} \in \overline{\mathcal{B}}_{C}(\mathcal{A})$. Using the fact that $\mathcal{P}$ is an $(M, C)$-quasigeodesic on the intervals $[0, T],[0, s]$, and $[s, T]$, one sees that $d(x, y) \geqslant\left(1 / M^{2}\right)(d(x, \mathcal{A})+d(y, \mathcal{A})-4 C)$. Therefore, if

$$
d(x, y)<\frac{1}{M^{2}} d(x, \mathcal{A})-\frac{4 C}{M^{2}},
$$

then $\mathcal{P}$ can not enter $\overline{\mathcal{B}}_{C}(\mathcal{A})$. This would contradict the constricting property, unless $d^{\pi}(x, y) \leqslant C$. Therefore, $\pi$ is $\left(M^{2}, \max \left\{C, 4 C / M^{2}\right\}\right)$-contracting.

Lemma 2.8. Let $\pi: \mathcal{X} \rightarrow \mathcal{A}$ be a $C$-coarse map that is $(1, C)$-PS-constricting. For all $x \in \mathcal{X}$ and all $r \geqslant 0$, we have

$$
\{a \in \mathcal{A} \mid d(x, a) \leqslant d(x, \mathcal{A})+r\} \subset\{a \in \mathcal{A} \mid d(a, \pi(x)) \leqslant r+5 C\} .
$$

In particular, setting $r=0$ shows that closest point projection to $\mathcal{A}$ is coarsely well defined and coarsely equivalent to $\pi$.

\footnotetext{
${ }^{3}$ Sisto [2011] calls this property " $\mathcal{P S}$-contracting". We change the name to avoid conflict with the better established "contracting" terminology of Definition 2.2.
} 
Proof. For $x \in \mathcal{X}$ and $r \geqslant 0$, let $a \in \mathcal{A}$ be a point such that $d(x, a) \leqslant d(x, \mathcal{A})+r$. Let $\mathcal{P}$ be a $(1, C)$-quasigeodesic from $x$ to $a$ in $\mathcal{P S}$. If $d(a, \pi(x))>2 C$, then $d^{\pi}(a, x)>C$, so there is a point $z \in \mathcal{P} \cap \overline{\mathcal{B}}_{C}(\pi(x))$. Now,

$$
d(x, z)+C \geqslant d(x, \pi(x)) \geqslant d(x, \mathcal{A}) \geqslant d(x, a)-r .
$$

Since $\mathcal{P}$ is a $(1, C)$-quasigeodesic, $d(x, a) \geqslant d(x, z)+d(z, a)-3 C$. As a result, $d(z, a) \leqslant r+4 C$, and $d(a, \pi(x)) \leqslant r+5 C$.

Proposition 2.9. Let $\pi: \mathcal{X} \rightarrow \mathcal{A}$. The following are equivalent:

(1) $\pi$ is strongly constricting.

(2) $\pi$ is constricting for some minimizing path system.

(3) $\pi$ is strongly contracting.

(4) $\pi$ has the bounded geodesic image property and $d(x, \pi(x))-d(x, \mathcal{A})$ is uniformly bounded.

Proof. The fact that (1) implies (2) is immediate.

Suppose $\pi$ is $(1, C)-\mathcal{P S}$-constricting for a minimizing path system $\mathcal{P S}$ consisting of $(1, C)$-quasigeodesics. Lemma 2.7 shows $\pi$ is $\left(1, C^{\prime}\right)$-contracting. By Lemma $2.8, \pi$ is coarsely a closest point projection, so $d(x, \pi(x))-d(x, \mathcal{A})$ is uniformly bounded. Thus, (2) implies (3).

Now suppose $\pi$ is $(1, C)$-contracting and $d(x, \pi(x))-d(x, \mathcal{A}) \leqslant C$ for all $x \in \mathcal{X}$. Take any geodesic $\mathcal{L}:[0, T] \rightarrow \mathcal{X}$. If $d^{\pi}\left(\mathcal{L}_{0}, \mathcal{L}_{T}\right)>10 C$ then $\mathcal{L} \cap \mathcal{B}_{3 C}(\mathcal{A}) \neq \varnothing$, as in Lemma 2.4. Let $t=t_{0}, t_{1}$ be the first and last times, respectively, such that $d\left(\mathcal{L}_{t}, \mathcal{A}\right) \leqslant 3 C$. By Lemma $2.4, d^{\pi}\left(\mathcal{L}_{0}, \mathcal{L}_{t_{0}}\right) \leqslant 10 C$. Thus,

$$
d\left(\pi\left(\mathcal{L}_{0}\right), \mathcal{L}_{t_{0}}\right) \leqslant d^{\pi}\left(\mathcal{L}_{0}, \mathcal{L}_{t_{0}}\right)+d\left(\pi\left(\mathcal{L}_{t_{0}}\right), \mathcal{L}_{t_{0}}\right) \leqslant 14 C .
$$

The same argument shows that $d\left(\pi\left(\mathcal{L}_{T}\right), \mathcal{L}_{t_{1}}\right) \leqslant 14 C$, so $\pi$ is $(1,14 C)$-constricting for the path system of all geodesics. Thus, (3) implies (1).

Finally, (3) is equivalent to (4) by Lemma 2.4.

2B. Additional properties of contracting and constricting maps. We establish some properties of contracting and constricting maps that will be useful in the sequel.

Lemma 2.10. If $\pi$ is a $(1, C)$-strongly constricting $C$-coarse map and $d^{\pi}(x, y)>C$, then $d(x, y) \geqslant d(x, \pi(x))+d^{\pi}(x, y)+d(\pi(y), y)-6 C$.

Proof. Let $\mathcal{L}$ be a geodesic from $x$ to $y$; by strong constriction, there exist $s$ and $t$ such that $d\left(\mathcal{L}_{s}, \pi(x)\right) \leqslant C$ and $d\left(\mathcal{L}_{t}, \pi(y)\right) \leqslant C$. The lemma follows from the triangle inequality and the fact that $\pi(x)$ and $\pi(y)$ have diameter at most $C$.

Lemma 2.11. If $\pi: \mathcal{X} \rightarrow \mathcal{A}$ is strongly constricting, then it is coarsely 1-Lipschitz. 
Proof. Let $\pi$ be a $C$-coarse map that is $(1, C)$-constricting on the path system of geodesics. Let $x_{0}$ and $x_{1}$ be arbitrary points, and let $\mathcal{L}$ be a geodesic from $x_{0}$ to $x_{1}$. If $d^{\pi}\left(x_{0}, x_{1}\right)>4 C$ then $\mathcal{L} \cap \mathcal{B}_{C}\left(x_{i}\right) \neq \varnothing$ for each $i$, which implies that $d\left(x_{0}, x_{1}\right) \geqslant d\left(x_{0}, \pi\left(x_{0}\right)\right)+d^{\pi}\left(x_{0}, x_{1}\right)+d\left(\pi\left(x_{1}\right), x_{1}\right)-8 C$. Thus, for all $x_{0}$ and $x_{1}$, we have $d^{\pi}\left(x_{0}, x_{1}\right) \leqslant d\left(x_{0}, x_{1}\right)+8 C$.

Lemma 2.12. Let $\pi: \mathcal{X} \rightarrow \mathcal{A}$ be an $(M, C)$-contracting $C$-coarse map such that $d(x, \pi(x))-d(x, \mathcal{A}) \leqslant C$ for all $x \in \mathcal{X}$. Fix $K \geqslant 1$. For all sufficiently large $D$, there exists a $T_{\max }$ such that if $\mathcal{Q}:[0, T] \rightarrow \mathcal{X}$ is a $(K, K)$-quasigeodesic with $d\left(\mathcal{Q}_{0}, A\right)=D=d\left(\mathcal{Q}_{T}, \mathcal{A}\right)$ and $\mathcal{Q} \cap \mathcal{B}_{D}(\mathcal{A})=\varnothing$ then $T \leqslant T_{\max }$.

Proof. Let $D>M\left(K^{2} C+C+K\right)$. Let $t_{0}:=0$ and let $t_{i+1}$ be the last time that $d\left(\mathcal{Q}_{t_{i}}, \mathcal{Q}_{t_{t+1}}\right)=(1 / M) d\left(\mathcal{Q}_{t_{i}}, \mathcal{A}\right)-C$, provided that $t_{i+1}<T$. This subdivides [0,T] into at most $1+(T K) /(D / M-C-K)$ intervals $\left[t_{0}, t_{1}\right], \ldots,\left[t_{k}, T\right]$, each of which has endpoints whose $\pi$-images are distance at most $C$ apart.

Since $\mathcal{Q}$ is a quasigeodesic, $T \leqslant K d\left(\mathcal{Q}_{0}, \mathcal{Q}_{T}\right)+K^{2}$. On the other hand:

$$
d\left(\mathcal{Q}_{0}, \mathcal{Q}_{T}\right) \leqslant 2 D+2 C+d^{\pi}\left(\mathcal{Q}_{0}, \mathcal{Q}_{T}\right) \leqslant 2 D+2 C+C\left(1+\frac{T K}{\frac{D}{M}-C-K}\right)
$$

Combined with the condition on $D$, this yields an upper bound on $T$.

Corollary 2.13. If $\pi: \mathcal{X} \rightarrow \mathcal{A}$ is contracting and $d(x, \pi(x))-d(x, \mathcal{A})$ is uniformly bounded, then for all $M \geqslant 1$ and $D \geqslant 0$ there exists a $K$ such that every $(M, M)$ quasigeodesic with endpoints at distance at most $D$ from $\mathcal{A}$ is contained in $\overline{\mathcal{B}}_{K}(\mathcal{A})$. In particular, if $\mathcal{A}$ is a quasigeodesic, then it is Morse.

Lemma 2.14. Let $\mathcal{Q}: \mathbb{R} \rightarrow \mathcal{X}$ be a quasigeodesic, and let $\pi: \mathcal{X} \rightarrow \mathcal{Q}$ be a strongly contracting projection. For all $D \geqslant 0$, there exists a $K$ such that if $\mathcal{P}:[0, T] \rightarrow \mathcal{X}$ is a geodesic and $t_{0}$ and $t_{1}$ are such that $d\left(\mathcal{P}_{0}, \mathcal{Q}_{t_{0}}\right) \leqslant D$ and $d\left(\mathcal{P}_{T}, \mathcal{Q}_{t_{1}}\right) \leqslant D$, then $\mathcal{Q}_{\left[t_{0}, t_{1}\right]} \subset \overline{\mathcal{B}}_{K}(\mathcal{P})$.

Proof. By Proposition $2.9, \pi$ is strongly constricting, so $\mathcal{P}$ passes close to every point in $\pi(\mathcal{P})$. Let $i$ and $j$ be numbers in the domain of $\mathcal{P}$, with $0<j-i \leqslant 1$. Let $s_{i}$ and $s_{j}$ be such that $\mathcal{Q}_{s_{i}} \in \pi\left(\mathcal{P}_{i}\right)$ and $\mathcal{Q}_{s_{j}} \in \pi\left(\mathcal{P}_{j}\right)$. Then $s_{i}$ and $s_{j}$ are boundedly far apart, since $\pi$ is coarsely 1-Lipschitz, by Lemma 2.11, and $\mathcal{Q}$ is a quasigeodesic. Therefore, the diameter of $\mathcal{Q}_{\left[s_{i}, s_{j}\right]}$ is bounded, and we have already noted that $\mathcal{Q}\left(s_{i}\right)$ and $\mathcal{Q}\left(s_{j}\right)$ are close to $\mathcal{P}$, since they are in the image of $\pi$.

Lemma 2.15. Let $\mathcal{A}$ and $\mathcal{A}^{\prime}$ be coarsely equivalent subsets of $\mathcal{X}$. Let $\sigma: \mathcal{A} \rightarrow \mathcal{A}^{\prime}$ and $\bar{\sigma}: \mathcal{A}^{\prime} \rightarrow \mathcal{A}$ be $C$-coarse maps such that $d(a, \sigma(a)) \leqslant C$ for all $a \in \mathcal{A}$ and $d\left(a^{\prime}, \bar{\sigma}\left(a^{\prime}\right)\right) \leqslant C$ for all $a^{\prime} \in \mathcal{A}^{\prime}$. Then, $\pi_{\mathcal{A}}: \mathcal{X} \rightarrow \mathcal{A}$ is strongly contracting if and only if $\pi_{\mathcal{A}^{\prime}}:=\sigma \circ \pi_{\mathcal{A}}: \mathcal{X} \rightarrow \mathcal{A}^{\prime}$ is strongly contracting. 
Proof. Suppose $\pi_{\mathcal{A}}$ is $(1, C)$-contracting and $d(x, \pi(x))-d(x, \mathcal{A}) \leqslant C$ for all $x \in \mathcal{X}$. If $d(x, y) \leqslant d\left(x, \mathcal{A}^{\prime}\right)-2 C \leqslant d(x, \mathcal{A})-C$, then $d_{\mathcal{A}^{\prime}}^{\pi}(x, y) \leqslant d_{\mathcal{A}}^{\pi}(x, y)+2 C \leqslant 3 C$, so $\pi_{\mathcal{A}^{\prime}}$ is $(1,3 C)$-contracting.

Take a point $x$ and let $a^{\prime} \in \mathcal{A}^{\prime}$ such that $d\left(x, \mathcal{A}^{\prime}\right)=d\left(x, a^{\prime}\right)$. Then,

$$
d\left(x, \bar{\sigma}\left(a^{\prime}\right)\right)-C \leqslant d\left(x, a^{\prime}\right) \leqslant d\left(x, \pi_{\mathcal{A}^{\prime}}(x)\right) \leqslant d\left(x, \pi_{\mathcal{A}}(x)\right)+2 C,
$$

so $d\left(x, \bar{\sigma}\left(a^{\prime}\right)\right) \leqslant d(x, \mathcal{A})+3 C$. By Proposition $2.9, \pi_{\mathcal{A}}$ is strongly constricting, so by Lemma 2.8 , there is a constant $D$ such that $d\left(\pi_{\mathcal{A}}(x), \bar{\sigma}\left(a^{\prime}\right)\right) \leqslant 3 C+D$. Thus, $\pi_{\mathcal{A}^{\prime}}$ is $(5 C+D)$-coarsely a closest point projection, hence, strongly contracting.

Lemma 2.16. Let $\pi: \mathcal{X} \rightarrow \mathcal{A}$ be strongly constricting. There exists a number $K$ such that if $d(\mathcal{A}, g \mathcal{A})>K$ then $\operatorname{diam} \pi(g \mathcal{A})$ is bounded, independent of $g$.

Proof. Let $\pi$ be $(1, C)$-strongly constricting. By Proposition $2.9, \pi$ is strongly contracting, so by Corollary 2.13 there is a constant $K$ such that a geodesic with endpoints in $\mathcal{A}$ stays in the $(K-C)$-neighborhood of $\mathcal{A}$. Therefore, a geodesic with endpoints in $g \mathcal{A}$ stays in $\overline{\mathcal{B}}_{K-C}(g \mathcal{A})$. Choose $x \in g \mathcal{A}$ such that $d(x, \mathcal{A})=d(g \mathcal{A}, \mathcal{A})$. For all $y \in g \mathcal{A}$, if $d^{\pi}(x, y)>C$, then a geodesic from $x$ to $y$ passes within distance $C$ of $\pi(x)$ and $\pi(y)$. This means $\overline{\mathcal{B}}_{C}(\mathcal{A}) \cap \overline{\mathcal{B}}_{K-C}(g \mathcal{A}) \neq \varnothing$, so $d(\mathcal{A}, g \mathcal{A}) \leqslant K$. Thus, if $d(\mathcal{A}, g \mathcal{A})>K$, then $d^{\pi}(x, y) \leqslant C$, so diam $\pi(g \mathcal{A}) \leqslant 2 C$.

2C. Strongly contracting elements. We have defined contraction and constriction for maps. We now give definitions for group elements:

Definition 2.17. An element $h \in G$ is called contracting, with respect to $G \curvearrowright \mathcal{X}$, if $i \mapsto h^{i} . o$ is a quasigeodesic and if there exists a subset $\mathcal{A} \subset \mathcal{X}$ on which $\langle h\rangle$ acts cocompactly and a map $\pi: \mathcal{X} \rightarrow \mathcal{A}$ that is contracting.

An element $h \in G$ is called constricting, with respect to $G \curvearrowright \mathcal{X}$, if $i \mapsto h^{i} . o$ is a quasigeodesic and if there exists a subset $\mathcal{A} \subset \mathcal{X}$ on which $\langle h\rangle$ acts cocompactly, a $G$-invariant path system $\mathcal{P S}$, and a map $\pi: \mathcal{X} \rightarrow \mathcal{A}$ that is $\mathcal{P S}$-constricting.

An element is strongly contracting or strongly constricting if the projection $\pi$ is, respectively, strongly contracting or strongly constricting.

For $\pi$ and $\mathcal{A}$ as in the definition, Proposition 2.9 says $\pi$ is strongly contracting if and only if it is strongly constricting. Thus, Lemma 2.8 says closest point projection to $\mathcal{A}$ is coarsely well defined and coarsely equivalent to $\pi$. Lemma 2.15 says that the choice of the set $\mathcal{A}$ only affects the constants of strong contraction. It follows that an element $h$ is strongly contracting if and only if $i \mapsto h^{i} . o$ is a quasigeodesic and closest point projection to $\langle h\rangle . o$ is strongly contracting. In the remainder of this section we produce more finely tailored choices for $\mathcal{A}$ and $\pi$. In particular, we would like $\pi$ to be compatible with the group action; see Remark 2.22. 
Proposition 2.18 (compare [Dahmani et al. 2011, Lemma 6.5]). Let $G$ be a finitely generated group, and let $\mathcal{X}$ be a $G$-space. Let $h \in G$ be an infinite order element. If there exists a strongly constricting $\pi: \mathcal{X} \rightarrow\langle h\rangle . o$, then $E(h)=H$, where

$$
H:=\{g \in G \mid g\langle h\rangle . o \text { is coarsely equivalent to }\langle h\rangle . o\} .
$$

Proof. $H$ is a group containing every finite index supergroup of $\langle h\rangle$. Let $D$ be the constant of Lemma 2.16, and let $S:=\{g \in \mathcal{G} \mid d(g\langle h\rangle . o,\langle h\rangle . o) \leqslant D\}$. Then, Lemma 2.16 implies $H \subset S$. Since $G \curvearrowright \mathcal{X}$ is properly discontinuous, $S$ is contained in finitely many $h$-orbits, so $\langle h\rangle<H$ has finite index. Therefore, $E(h)$ exists and is equal to $H$.

Definition 2.19. If $h$ is a strongly contracting element, define the (quasi-)axis of $h$, with respect to the basepoint $o$, to be $\mathcal{H}:=E(h)$. $o$.

Lemma 2.20. If $h$ is a strongly contracting element, then there exists an $E(h)-$ equivariant, strongly contracting coarse map $\pi_{\mathcal{H}}: \mathcal{X} \rightarrow \mathcal{H}$.

Proof. By Proposition 2.9, Lemma 2.8, and Lemma 2.15 any choice of closest point projection map to $\mathcal{H}$ is strongly contracting and coarsely $E(h)$-equivariant, so, by Lemma 1.1, we can replace it by a coarsely equivalent, $E(h)$-equivariant coarse map, which will still be strongly contracting, by Lemma 2.15.

Definition 2.21. From the projection $\pi_{\mathcal{H}}$ of Lemma 2.20, define strongly contracting projections onto each translate of $\mathcal{H}$ by $\pi_{g \mathcal{H}}: \mathcal{X} \rightarrow g \mathcal{H}, x \mapsto g . \pi_{\mathcal{H}}\left(g^{-1} . x\right)$.

If $g^{\prime} \mathcal{H}=g \mathcal{H}$ then $g^{-1} g^{\prime} \in E(h)$, so Lemma 2.20 implies that $\pi_{g^{\prime} \mathcal{H}}(x)=\pi_{g \mathcal{H}}(x)$ for all $x \in \mathcal{X}$.

Remark 2.22. The projections of Definition 2.21 satisfy $g \cdot \pi_{\mathcal{H}}(x)=\pi_{g \mathcal{H}}(g \cdot x)$ for all $x \in \mathcal{X}$ and $g \in G$.

2D. Strongly contracting elements and the projection axioms. Let $h \in G$ be a strongly contracting element with respect to $G \curvearrowright \mathcal{X}$. Let $\mathcal{H}$ be a quasi-axis of $h$ defined in Definition 2.19. We wish to apply Theorem 1.11 to the collection of $G$-translates of $\mathcal{H}$ with the projections of Definition 2.21. To see that the hypotheses of the theorem are satisfied, we first embed $\mathcal{H}$ into a geodesic metric space and then verify the projection axioms of Definition 1.10.

Choose representatives $1=g_{0}, \ldots, g_{n-1}$ for $\langle h\rangle \backslash E(h)$, so that for each $i$ we have $d\left(g_{i} . o, o\right)=\min _{g \in\langle h\rangle g_{i}} d(g . o, o)$. Let $g_{n}:=h$. Let $\hat{H}$ be the Cayley graph of $E(h)$ with respect to the generating set $\left\{g_{1}, \ldots, g_{n}\right\}$. The graph $\hat{H}$ becomes a geodesic metric space by assigning each edge length one, and it is a quasitree since $E(h)$ is virtually cyclic.

Choose representatives $1=f_{0}, f_{1}, \ldots$ for $G / E(h)$. Let $\mathbb{Y}$ be a disjoint union of copies of $\hat{H}$, one for each coset $f_{i} E(h) \in G / E(h)$, denoted $f_{i} \hat{H}$. The orbit 
map $f_{i} \hat{H} \rightarrow f_{i} \mathcal{H}$, defined by $f_{i} e \mapsto f_{i} e . o$, is a quasi-isometric embedding, so its inverse $\phi_{f_{i} \mathcal{H}}: f_{i} \mathcal{H} \rightarrow f_{i} \hat{H}$ is a coarse map that is a quasi-isometry. Define $\pi_{f_{i} \hat{H}}\left(f_{j} \hat{H}\right):=\phi_{f_{i}}\left(\pi_{f_{i} \mathcal{H}}\left(f_{j} \mathcal{H}\right)\right)$. Since $\phi_{f_{i}}$ is a quasi-isometry, it suffices to check the projection axioms on translates of $\mathcal{H}$ in $\mathcal{X}$.

Lemma 2.23 (Axiom (P0)). There is a uniform bound on the diameter of $\pi_{\mathcal{H}}(g \mathcal{H})$ for $g \notin E(h)$.

Proof. Let $\pi_{\mathcal{H}}: \mathcal{X} \rightarrow \mathcal{H}$ be $\left(1, C^{\prime}\right)$-strongly constricting. Let $\mathcal{Q}: \mathbb{R} \rightarrow \mathcal{H}$ be an $\left(M, C^{\prime \prime}\right)$-quasigeodesic parametrization that agrees with $i \mapsto h^{i} . o$ on the integers. Replace $C^{\prime}$ and $C^{\prime \prime}$ by $C:=\max \left\{C^{\prime}, C^{\prime \prime}\right\}$.

Let $D:=\operatorname{diam}\langle h\rangle \backslash \mathcal{H}$. Let $K$ be large enough such that whenever $\mathcal{P}$ is a geodesic with $d\left(\mathcal{P}_{s_{0}}, \mathcal{Q}_{t_{0}}\right) \leqslant C$ and $d\left(\mathcal{P}_{s_{1}}, \mathcal{Q}_{t_{1}}\right) \leqslant C$, we have $\mathcal{P}_{\left[s_{0}, s_{1}\right]} \subset \overline{\mathcal{B}}_{K}\left(\mathcal{Q}_{\left[t_{0}, t_{1}\right]}\right)$ and $\mathcal{Q}_{\left[s_{0}, s_{1}\right]} \subset \overline{\mathcal{B}}_{K}\left(\mathcal{P}_{\left[t_{0}, t_{1}\right]}\right)$, as in Corollary 2.13 and Lemma 2.14.

Suppose $g \notin E(h)$. For a pair of points $x_{0}, x_{1} \in g \mathcal{H}$, take $t_{0}$ and $t_{1}$ such that $\mathcal{Q}_{t_{i}} \in \pi_{\mathcal{H}}\left(x_{i}\right)$ for each $i$. Let $\mathcal{P}$ be a geodesic connecting $x_{0}$ to $x_{1}$. If $d_{\mathcal{H}}^{\pi}\left(x_{0}, x_{1}\right)>C$, then for each $i$ there exists $s_{i}$ such that $d\left(\mathcal{P}_{s_{i}}, \mathcal{Q}_{t_{i}}\right) \leqslant C$.

Now, $\mathcal{Q}_{\left[t_{0}, t_{1}\right]}$ is $K$-close to $\mathcal{P}_{\left[s_{0}, s_{1}\right]}$, which in turn is $K$-close to a subinterval of $g \mathcal{H}$. Therefore, for each integer $i \in\left[t_{0}, t_{1}\right]$ there is an integer $\alpha_{i}$ such that $d\left(h^{i} . o, g h^{\alpha_{i}} g^{-1} . o\right) \leqslant 2 K+D$.

If, for some $i \neq j$, the equation $h^{-i} g h^{\alpha_{i}} g^{-1} . o=h^{-j} g h^{\alpha_{j}} g^{-1} . o$ is satisfied, then $h^{j-i}=g h^{\alpha_{j}-\alpha_{i}} g^{-1}$, which implies $\langle h\rangle$ and $\left\langle g h g^{-1}\right\rangle$ are commensurable. However, this would imply $g \in E(h)$, contrary to hypothesis. Therefore, for each integer $i$ in $\left[t_{0}, t_{1}\right]$ we get a distinct point $h^{-i} g h^{\alpha_{i}} g^{-1} . o \in \overline{\mathcal{B}}_{2 K+D}(o)$. Since the action of $G$ is properly discontinuous, the number of orbit points in $\overline{\mathcal{B}}_{2 K+D}(o)$ is finite, so $\operatorname{diam} \pi_{\mathcal{H}}(g \mathcal{H})$ is bounded, independent of $g$.

Lemma 2.24 (Axiom (P1)). For all sufficiently large $\xi$ and for any $X, Y, Z \in \mathbb{Y}$, at most one of $d_{X}^{\pi}(Y, Z), d_{Y}^{\pi}(X, Z)$, and $d_{Z}^{\pi}(X, Y)$ is greater than $\xi$.

Proof. Suppose $\pi_{Y}$ is $(1, C)$-strongly constricting. Let $\xi^{\prime}$ be the constant from Lemma 2.23. Let $\xi \geqslant 2 \xi^{\prime}+14 C$. Suppose that $d_{X}^{\pi}(Y, Z)>\xi$. We show $d_{X}^{\pi}(Y, Z) \leqslant \xi$; the inequality $d_{Z}^{\pi}(X, Y) \leqslant \xi$ follows by a similar argument.

Take any point $z \in Z$, and let $y \in Y$ be a point such that $d(z, y)=d(z, Y)$. Let $\mathcal{L}:[0, T] \rightarrow \mathcal{X}$ be a geodesic from $z$ to $y$. For every point of $\mathcal{L}, y$ is the closest point of $Y$. By Lemma 2.8, $\pi_{Y}(\mathcal{L}) \subset \overline{\mathcal{B}}_{5 C}(y)$. Now, $d_{X}^{\pi}(Y, Z)>\xi$ implies $d_{X}^{\pi}\left(\mathcal{L}_{0}, \mathcal{L}_{T}\right)>C$, so there are $z^{\prime} \in \mathcal{L}$ and $x \in X$ with $d\left(x, z^{\prime}\right) \leqslant D$. By Lemma 2.11, $\pi_{Y}$ is $8 C$-coarsely 1 -Lipschitz, which means $d_{Y}^{\pi}\left(x, z^{\prime}\right) \leqslant 9 C$. Thus,

$$
d_{Y}^{\pi}(X, Z) \leqslant 2 \xi^{\prime}+d_{Y}^{\pi}(x, z) \leqslant 2 \xi^{\prime}+5 C+d_{Y}^{\pi}\left(x, z^{\prime}\right) \leqslant 2 \xi^{\prime}+14 C \leqslant \xi .
$$

Lemma 2.25 (Axiom (P2)). For all sufficiently large $\xi$ and for all $X, Y \in \mathbb{Y}$, the set $\left\{V \in \mathbb{Y} \mid d_{V}^{\pi}(X, Y)>\xi\right\}$ is finite. 
Proof. Let $\xi^{\prime}$ be the constant of Lemma 2.23. Suppose $\pi_{\mathcal{H}}$ is $(1, C)$-strongly constricting. Let $\xi>C+2 \xi^{\prime}$. Take arbitrary $X, Y \in \mathbb{Y}$, and let $\mathcal{L}$ be a geodesic from some point in $\pi_{X}(Y)$ to some point in $\pi_{Y}(X)$. If $d_{V}^{\pi}(X, Y)>\xi$, then $d_{V}^{\pi}\left(\mathcal{L}_{0}, \mathcal{L}_{T}\right)>C$, so $\mathcal{L}$ comes within distance $C$ of $V$. By proper discontinuity of the action, there are only finitely many elements of $\varangle$ that come within distance $C$ of the finite geodesic $\mathcal{L}$.

Notation 2.26. Let $\mathcal{Y}$ be the quasitree produced by Theorem 1.11 from $\mathbb{Y}$, and let $\star \in \mathcal{Y}$ be the vertex corresponding to $o \in \mathcal{X}$. Furthermore, let $\hat{\pi}_{g \hat{H}}: \mathcal{Y} \rightarrow g \hat{H}$ be closest point projection to the isometrically embedded copy of $g \hat{H}$ in $\mathcal{Y}$, which the theorem says coarsely agrees with $\pi_{g \hat{H}}$.

Definition 2.27. Define uniform quasi-isometric embeddings $\phi_{g \mathcal{H}}: g \mathcal{H} \rightarrow \mathcal{Y}$ for each translate $g \mathcal{H}$ of $\mathcal{H}$ by sending $g \mathcal{H}$ to $f_{i} \hat{H}$ via $\phi_{f_{i}}$, where $g \in f_{i} E(h)$, and postcomposing by the isometric embedding of $f_{i} \hat{H}$ into $\mathcal{Y}$ provided by Theorem 1.11.

Proposition 2.28. If there is a strongly contracting element for $G \curvearrowright \mathcal{X}$, then $G$ has nonzero growth exponent.

Proof. [Bestvina et al. 2014, Proposition 3.23] says that $G$ contains a free subgroup, so it has exponential growth.

\section{Abundance of strongly contracting elements}

In this section we show that strongly contracting elements are abundant.

Proposition 3.1. If $G$ contains a strongly contracting element for $G \curvearrowright \mathcal{X}$, then so does every infinite normal subgroup.

In effect, the proposition reduces the problem of growth tightness for arbitrary quotients of $G$ to quotients by the normal closure of a strongly contracting element.

Given a strongly contracting element $h \in \mathcal{G}$ and an infinite normal subgroup $\Gamma$ of $G$ we find an element $g \in \Gamma$ such that $f:=g h^{n} g^{-1} h^{-n} \in \Gamma$ is strongly contracting for all sufficiently large $n$. To prove $f$ is strongly contracting we follow a standard strategy by showing that an axis for $f$ has "long" $(\asymp n)$ segments in contracting sets, separated by "short" ( $=d(o, g . o))$ hops between such segments. For each $x \in \mathcal{X}$ there is, coarsely, a unique one of these segments such that the projection of $x$ transitions from landing at the end of the segment to landing at the beginning of the segment. We use this transition point to define the projection to the $f$-axis, and verify that this projection is strongly contracting.

We first prove some preliminary lemmas.

Lemma 3.2. Let $h \in G$ be an infinite order element and let $\pi: \mathcal{X} \rightarrow\langle h\rangle$.o be a contracting coarse map such that $d(x, \pi(x))-d(x, \mathcal{A})$ is uniformly bounded. Then, $i \mapsto h^{i}$.o is a quasigeodesic. 
Proof. Take any $\alpha<\beta$ in $\mathbb{Z}$. By the triangle inequality, $d\left(h^{\alpha} . o, h^{\beta} . o\right) \stackrel{*}{\prec}(\beta-\alpha)$. We now prove the opposite inequality. Let $\mathcal{L}:[0, T] \rightarrow \mathcal{X}$ be a geodesic from $h^{\alpha}$.o to $h^{\beta}$.o. By Corollary 2.13, there exists a $D$ such that for every $i \in[0, T] \cap \mathbb{Z}$ there exists an $\alpha \leqslant \alpha_{i} \leqslant \beta$ such that $d\left(\mathcal{L}_{i}, h^{\alpha_{i}} . o\right) \leqslant D$. Since the action of $G$ on $\mathcal{X}$ is properly discontinuous, there exists a maximum $\gamma$ such that $d\left(o, h^{\gamma} . o\right) \leqslant 2 D+1$, so $\alpha_{i+1}-\alpha_{i} \leqslant \gamma$ for all $i$. Setting $\alpha_{0}:=\alpha$ and $\alpha_{\lceil T\rceil}:=\beta$, we have

$$
\beta-\alpha=\sum_{i=0}^{\lceil T\rceil-1} \alpha_{i+1}-\alpha_{i} \leqslant \gamma\lceil T\rceil \leqslant \gamma\left(d\left(h^{\alpha} . o, h^{\beta} . o\right)+1\right) .
$$

Fix a strongly contracting element $h$, and let $\mathcal{Y}$ be the quasitree of Notation 2.26, with bottleneck constant $\Delta$.

Lemma 3.3. There exists $K \geqslant 0$ such that $d_{\mathcal{H}}^{\pi}\left(o, g_{1} . o\right)-d_{\mathcal{H}}^{\pi}\left(g_{1} . o, g_{0} . o\right) \geqslant K$ implies that $g_{0} \star \star$ and $g_{1} . \star$ are contained in the same component of $\mathcal{Y} \backslash \overline{\mathcal{B}}_{\Delta}(\star)$.

Proof. Let $D:=\operatorname{diam}\langle h\rangle \backslash \hat{H}$ in $\mathcal{Y}$. For each $i \in\{0,1\}$, choose an $m_{i}$ such that $d\left(h^{m_{i}} \cdot \star, \hat{\pi}_{\hat{H}}\left(g_{i} . \star\right)\right) \leqslant D$. Choose a geodesic $\mathcal{L}$ from $\star$ to $h . \star$. Take $M>0$ such that $h^{m} \cdot \mathcal{L} \cap \overline{\mathcal{B}}_{\Delta}(\star)=\varnothing$ when $|m| \geq M$.

For each $i,\left|m_{i}\right| \asymp d\left(h^{m_{i}} \star \star, \star\right) \succ K$, so for sufficiently large $K$, we have the lower bound $d\left(h^{m_{i}} . \star, \star\right)>2 \Delta+D$ and $\left|m_{i}\right|>M$. Furthermore, $m_{0}$ and $m_{1}$ must have the same sign if $K$ is large enough: by Lemma 2.14, the interval of $\mathcal{H}$ between $h^{m_{0}} . o$ and $h^{m_{1}} . o$ stays close to a geodesic between $h^{m_{0}} . o$ and $h^{m_{1}} . o$, so if $m_{0}$ and $m_{1}$ have different signs, then

$$
\begin{aligned}
d_{\mathcal{H}}^{\pi}\left(g_{0} . o, g_{1} . o\right) & \doteq d\left(h^{m_{0}} . o, h^{m_{1}} . o\right) \\
& \doteq d\left(o, h^{m_{0}} . o\right)+d\left(o, h^{m_{1}} . o\right) \doteq d_{\mathcal{H}}^{\pi}\left(o, g_{0} . o\right)+d_{\mathcal{H}}^{\pi}\left(o, g_{1} . o\right) .
\end{aligned}
$$

However, $d_{\mathcal{H}}^{\pi}\left(g_{0} . o, g_{1} . o\right) \leqslant d_{\mathcal{H}}^{\pi}\left(o, g_{1} . o\right)-K$, so this would imply

$$
K \stackrel{ \pm}{\prec} d_{\mathcal{H}}^{\pi}\left(o, g_{0} . o\right) \stackrel{ \pm}{\prec}-K,
$$

which is false for sufficiently large $K$.

No geodesic between $g_{i} \star \star$ and $h^{m_{i}} \star \star$ enters $\overline{\mathcal{B}}_{\Delta}(\star)$, since this would imply:

$$
d\left(h^{m_{1}} . \star, \star\right) \leqslant 2 \Delta+D .
$$

For $\min \left\{m_{0}, m_{1}\right\} \leqslant m \leqslant \max \left\{m_{0}, m_{1}\right\}-1$, the geodesic $h^{m} \cdot \mathcal{L}$ stays outside $\overline{\mathcal{B}}_{\Delta}(\star)$ since $m_{0}$ and $m_{1}$ have the same sign and magnitude at least $M$, which implies that $|m| \geqslant M$. By concatenating such geodesics, we construct a path from $g_{0} \star \star$ to $g_{1} \star \star$ in $\mathcal{Y} \backslash \overline{\mathcal{B}}_{\Delta}(\star)$.

Corollary 3.4. There exists an $N>0$ such that for all $n \geqslant N$ the points $h^{n} . \star$ and $h^{N} . \star$ are in the same component of $\mathcal{Y} \backslash \overline{\mathcal{B}}_{\Delta}(\star)$. 
Proof. Take $N$ large enough so that $d_{\mathcal{H}}^{\pi}\left(o, h^{n} . o\right) \geqslant K+d(o, h . o)+2 C$ for all $n \geqslant N$. Then, $d_{\mathcal{H}}^{\pi}\left(o, h^{n+1} . o\right)-d_{\mathcal{H}}^{\pi}\left(h^{n} . o, h^{n+1} . o\right) \geqslant K$. Apply Lemma 3.3.

Definition 3.5. Call the component of $\mathcal{Y} \backslash \mathcal{B}_{\Delta}(g . \star)$ containing $g h^{n} . \star$ for all sufficiently large $n$ the $g h^{\infty}$ component and the component containing $g h^{-n}$. $\star$ for all sufficiently large $n$ the $g h^{-\infty}$ component.

Lemma 3.6. For some $K \geqslant 0$, suppose $g_{0}$ and $g_{1}$ are elements of $G$ such that $g_{0} \mathcal{H} \neq g_{1} \mathcal{H}$ and $d_{g_{0} \mathcal{H}}^{\pi}\left(g_{0} . o, g_{1} . o\right) \leqslant K$ and $d_{g_{1} \mathcal{H}}^{\pi}\left(g_{0} . o, g_{1} . o\right) \leqslant K$. Then, there exists an $N>0$ such that for all $n \geqslant N, \epsilon_{0}, \epsilon_{1} \in\{ \pm 1\}$, and $f_{0}, f_{1} \in\left\{g_{0}, g_{1}\right\}$,

- the balls $\overline{\mathcal{B}}_{\Delta}\left(f_{0} h^{\epsilon_{0} n / 2} . \star\right)$ and $\overline{\mathcal{B}}_{\Delta}\left(f_{1} h^{\epsilon_{1} n / 2} . \star\right)$ in $\mathcal{Y}$ are disjoint unless $f_{0}=f_{1}$ and $\epsilon_{0}=\epsilon_{1}$,

- $f_{0} . \star$ and $f_{1} \star \star$ are in the $f_{0} h^{-\epsilon_{0} \infty}$ component of $\mathcal{Y} \backslash \overline{\mathcal{B}}_{\Delta}\left(f_{0} h^{\epsilon_{0} n / 2} . \star\right)$, and

- $f_{0} h^{\epsilon_{0} n} . \star$ and $f_{0} h^{\epsilon_{0} n} f_{1}$. $\star$ are in the $f_{0} h^{\epsilon_{0} \infty}$ component of $\mathcal{Y} \backslash \overline{\mathcal{B}}_{\Delta}\left(f_{0} h^{\epsilon_{0} n / 2} . \star\right)$.

Proof. $\overline{\mathcal{B}}_{\Delta}\left(f_{0} h^{n / 2} . \star\right)$ and $\overline{\mathcal{B}}_{\Delta}\left(f_{0} h^{-n / 2} . \star\right)$ are disjoint for all sufficiently large $n$ since $i \mapsto h^{i} . \star$ is a quasigeodesic. In the other cases, $f_{0} \mathcal{H}$ and $f_{1} \mathcal{H}$ are distinct axes, so $f_{0} \hat{H}$ and $f_{1} \hat{H}$ are disjoint. For each $i \in\{0,1\}$, the bounds

$$
d_{f_{i} \mathcal{H}}^{\pi}\left(f_{i} . o, f_{1-i} . o\right) \leqslant K
$$

imply that the closest point projection $\hat{\pi}_{f_{i}} \hat{H}\left(f_{1-i} \hat{H}\right)$ of $f_{1-i} \hat{H}$ to $f_{i} \hat{H}$ is contained in a bounded neighborhood of $f_{i} . \star$. For any point $y . \star \in \overline{\mathcal{B}}_{\Delta}\left(f_{1} h^{\epsilon_{1} n / 2} . \star\right) \backslash f_{1} \hat{H}$, we have that $\hat{\pi}_{f_{1} \hat{H}}(y \hat{H})$ is $2 \Delta$-close to $f_{1} h^{\epsilon_{1} n / 2}$. $\star$. Therefore,

$$
d_{f_{1} \hat{H}}^{\pi}\left(f_{0} \hat{H}, y \hat{H}\right) \succsim d\left(f_{1} \cdot \star, f_{1} h^{\epsilon_{1} n / 2} . \star\right) \asymp n,
$$

so for $n$ sufficiently large we can make $d_{f_{1} \hat{H}}^{\pi}\left(f_{0} \hat{H}, y \hat{H}\right)$ larger then the constant $\xi$ of projection axiom (P1), which implies $d_{f_{0}}^{\pi} \hat{H}\left(f_{1} \hat{H}, y \hat{H}\right) \leqslant \xi$. On the other hand, $\overline{\mathcal{B}}_{\Delta}\left(f_{0} h^{\epsilon_{0} n / 2} . \star\right)$ projects close to $f_{0} h^{\epsilon_{0} n / 2} \star \star$ in $f_{0} \hat{H}$, so for large enough $n$ the balls have disjoint projections, which means the balls are disjoint.

For the second statement, suppose $N$ is large enough so that for all $n \geqslant N$ we have $d\left(o, h^{n / 2} . o\right) \geqslant K^{\prime}+K+2 C$, where $K^{\prime}$ is the constant of Lemma 3.3. Then,

$$
d_{f_{0} \mathcal{H}}^{\pi}\left(f_{0} h^{\epsilon_{0} n / 2} . o, f_{0} . o\right)-d_{f_{0} \mathcal{H}}^{\pi}\left(f_{0} . o, f_{1} . o\right) \geqslant K^{\prime},
$$

so Lemma 3.3 implies $f_{0} \star \star$ and $f_{1} . \star$ are in the same component of $\mathcal{Y} \backslash \overline{\mathcal{B}}_{\Delta}\left(f_{0} h^{\epsilon_{0} n / 2} \star \star\right)$. If, in addition, $N$ is at least twice the constant of Corollary 3.4, then this is the $f_{0} h^{-\epsilon_{0} \infty}$ component.

The proof of the third statement is similar. 


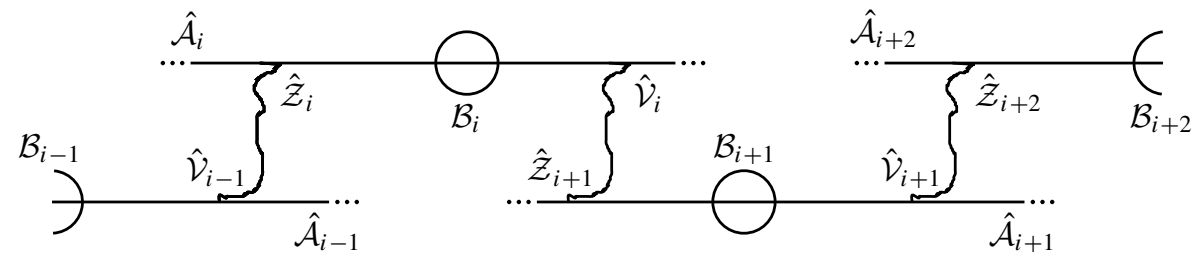

Figure 1. Disjoint balls in $\mathcal{Y}$.

Proof of Proposition 3.1. Strongly constricting is the same as strongly contracting, by Proposition 2.9, so suppose $h$ is a $(1, C)$-strongly constricting element. By Lemma 2.8 , there exists a $D$ such that $\pi_{\mathcal{H}}$ is $D$-coarsely equivalent to closest point projection. Recall that $D>C$. By Lemma 2.11, there exists a $D^{\prime}$ such that $\pi_{\mathcal{H}}$ is $D^{\prime}$-coarsely 1-Lipschitz.

Let $\Gamma$ be an infinite normal subgroup of $G$. Every infinite order element of $E(h)$ is strongly contracting, so if $\Gamma$ contains such an element then we are done. Otherwise, $\Gamma \cap E(h)$ is finite. Since $\Gamma$ is infinite, there exists an element $g \in \Gamma$ such that $g \notin E(h)$. We claim that for sufficiently large $n$ the element $f:=g h^{n} g^{-1} h^{-n} \in \Gamma$ is strongly constricting.

For brevity, let $f^{i+1 / 2}$ denote $f^{i} g h^{n}$. Let $\hat{\mathcal{A}}_{i}:=f^{i / 2} \hat{H}$ and $\mathcal{A}_{i}:=f^{i / 2} \mathcal{H}$. Define $\mathcal{B}_{0}:=\overline{\mathcal{B}}_{\Delta}\left(h^{n / 2} \star_{\star}\right), \mathcal{B}_{1}:=\overline{\mathcal{B}}_{\Delta}\left(f^{1 / 2} h^{-n / 2} . \star\right)$, and $\mathcal{B}_{2 k+i}:=f^{k} \mathcal{B}_{i}$ for $k \in \mathbb{Z}$. Let $\hat{\mathcal{Z}}_{i}:=f^{i / 2} h^{(-1)^{i} n} . \star \in \mathcal{Y}$ and $\mathcal{Z}_{i}:=f^{i / 2} h^{(-1)^{i} n} . o \in \mathcal{X}$. Let $\hat{\mathcal{V}}_{i}:=f^{i / 2} . \star \in \mathcal{Y}$ and $\mathcal{V}_{i}:=f^{i / 2} . o \in \mathcal{X}$. See Figure 1 .

By repeated applications of Lemma 3.6, for large enough $n$, the balls $\mathcal{B}_{i}$ are pairwise disjoint. There are two orbits of these balls under the $f$-action, so $f$ is an infinite order element. Furthermore, the balls are linearly ordered by separation, consistent with the subscripts, since for all $i$ we have that $\mathcal{B}_{j}$ is contained in the $f^{i / 2} h^{(-1)^{i+1} \infty}$ component of $\mathcal{Y} \backslash \mathcal{B}_{i}$ for all $j>i$, and in the $f^{i / 2} h^{(-1)^{i} \infty}$ component for all $j<i$.

For any $i$ and any $j<i-1$ the ball $\mathcal{B}_{i-1}$ separates $\hat{\mathcal{A}}_{j}$ from $\hat{\mathcal{A}}_{i}$ in $\mathcal{Y}$, so $\hat{\pi}_{\hat{\mathcal{A}}_{i}}\left(\hat{\mathcal{A}}_{j}\right)$ is contained in a bounded neighborhood of $\hat{\pi}_{\hat{\mathcal{A}}_{i}}\left(\hat{\mathcal{A}}_{i-1}\right)$, which in turn we know is contained in a bounded neighborhood of $\hat{\mathcal{Z}}_{i}$. Conversely, $\hat{\pi}_{\hat{\mathcal{A}}_{i}}\left(\hat{\mathcal{A}}_{j}\right)$ is contained in a bounded neighborhood of $\hat{\mathcal{V}}_{i}$ for $j>i$. Since $\hat{\pi}_{\hat{\mathcal{A}}_{i}}$ agrees with $\pi_{\mathcal{A}_{i}}$ up to bounded error, the same statements are true for the axes in $\mathcal{X}$. That is, there exists a $K$, independent of $n$, such that for all $i$,

- $d_{\mathcal{A}_{i}}^{\pi}\left(\mathcal{Z}_{i}, \mathcal{A}_{j}\right) \leqslant K$ if $j<i$, and

- $d_{\mathcal{A}_{i}}^{\pi}\left(\mathcal{V}_{i}, \mathcal{A}_{j}\right) \leqslant K$ if $j>i$.

Define $K^{\prime}:=2 K+C+2 D+D^{\prime}$.

Suppose that for some $x \in \mathcal{X}$ there exists an $i$ such that $d_{\mathcal{A}_{i}}^{\pi}\left(x, \mathcal{V}_{i}\right)>K^{\prime}$. Then for any $j>i$ we have $d\left(\pi_{\mathcal{A}_{i}}(x), \pi_{\mathcal{A}_{i}}\left(\mathcal{A}_{j}\right)\right)>D>C$. Let $y$ be a point of $\mathcal{A}_{j}$ closest 


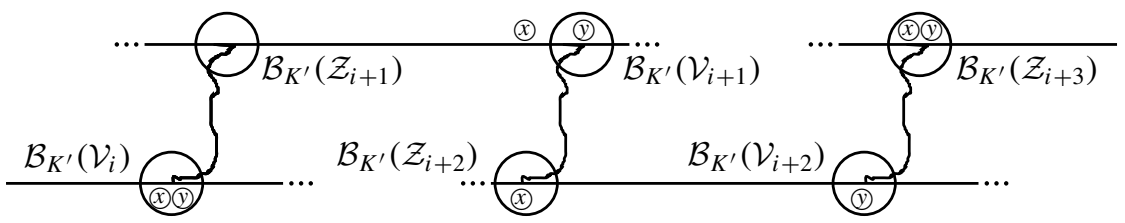

Figure 2. Projections $(x)$ of $x$ and (y) of $y$ to each axis.

to $x$. On any given geodesic from $x$ to $y$, there is a point $z \in \overline{\mathcal{B}}_{C+K}\left(\mathcal{V}_{i}\right)$, since $d_{\mathcal{A}_{i}}^{\pi}(x, y)>C$. Now $\pi_{\mathcal{A}_{j}}$ is $D$-coarsely equivalent to closest point projection, and $y$ is closest to both $x$ and $z$, so $d_{\mathcal{A}_{j}}^{\pi}(x, z) \leqslant 2 D$. However, $z$ is $(C+K)$-close to $\mathcal{V}_{i}$, and $d_{\mathcal{A}_{j}}^{\pi}\left(\mathcal{V}_{i}, \mathcal{Z}_{j}\right) \leqslant K$, so $d_{\mathcal{A}_{j}}^{\pi}\left(x, \mathcal{Z}_{j}\right) \leqslant 2 D+C+K+D^{\prime}+K=K^{\prime}$.

We have shown that $d_{\mathcal{A}_{i}}^{\pi}\left(x, \mathcal{V}_{i}\right)>K^{\prime}$ implies $d_{\mathcal{A}_{j}}^{\pi}\left(x, \mathcal{Z}_{j}\right) \leqslant K^{\prime}$ for all $j>i$. A similar argument shows that $d_{\mathcal{A}_{i}}^{\pi}\left(x, \mathcal{Z}_{i}\right)>K^{\prime}$ implies $d_{\mathcal{A}_{j}}^{\pi}\left(x, \mathcal{V}_{j}\right) \leqslant K^{\prime}$ for all $j<i$.

Assume that $n$ is large enough so that

$$
d_{\mathcal{A}_{0}}^{\pi}\left(\mathcal{Z}_{0}, \mathcal{V}_{0}\right)=d_{\mathcal{H}}^{\pi}\left(h^{n} . o, o\right)>2 K^{\prime}+2 C+2 D+d(o, g . o)
$$

Define $\mathcal{F}:=\bigcup_{i \in \mathbb{Z}}\left\{\mathcal{V}_{i}\right\}$. We wish to define $\pi_{\mathcal{F}}: \mathcal{X} \rightarrow \mathcal{F}$ by sending a point $x$ to the point $\mathcal{V}_{\alpha}$ where $\alpha$ is the greatest integer such that $d_{\mathcal{A}_{\alpha}}^{\pi}\left(x, \mathcal{V}_{\alpha}\right) \leqslant K^{\prime}$, but we must verify that such an $\alpha$ exists. Fix an $x \in \mathcal{X}$, and suppose that $\iota \in \mathbb{Z}$ is such that $d\left(x, \mathcal{A}_{\iota}\right)=\min _{j \in \mathbb{Z}} d\left(x, \mathcal{A}_{j}\right)$. Such an $\iota$ exists since the action is properly discontinuous. Suppose that $d_{\mathcal{A}_{l}}^{\pi}\left(x, \mathcal{V}_{\iota}\right) \leqslant K^{\prime}$. By the assumption on $n$, $d_{\mathcal{A}_{l}}^{\pi}\left(x, \mathcal{Z}_{l}\right)>K^{\prime}$, so $d_{\mathcal{A}_{j}}^{\pi}\left(x, \mathcal{V}_{l}\right) \leqslant K^{\prime}$ for all $j<\iota$. A brief computation shows that

$$
d_{\mathcal{A}_{l+1}}^{\pi}\left(x, \mathcal{Z}_{l+1}\right) \leqslant d\left(x, \mathcal{A}_{l+1}\right)+d(o, g . o)+K^{\prime}+2 C+D .
$$

By Lemma $2.8, d\left(\mathcal{Z}_{l+1}, \pi_{\mathcal{A}_{l+1}}(x)\right) \leqslant d(o, g . o)+K^{\prime}+2 C+2 D$, which, again by our assumption on $n$, implies $d_{\mathcal{A}_{l+1}}^{\pi}\left(x, \mathcal{V}_{\iota+1}\right)>K^{\prime}$. We conclude that $\alpha \leqslant \iota$. The previous paragraph then tells us that $d_{\mathcal{A}_{j}}^{\pi}\left(x, \mathcal{Z}_{j}\right) \leqslant K^{\prime}$ for all $j>\alpha+1$.

Now suppose $x$ and $y$ are points with $\pi_{\mathcal{F}}(x)=\mathcal{V}_{i}$ and $\pi_{\mathcal{F}}(y)=\mathcal{V}_{j}$ for $j>i+1$. Then for each $i+2 \leqslant k \leqslant j$, we have $d_{\mathcal{A}_{k}}^{\pi}(x, y) \geqslant d_{\mathcal{A}_{k}}^{\pi}\left(\mathcal{Z}_{k}, \mathcal{V}_{k}\right)-2 K^{\prime}>C$. Figure 2 depicts a situation with $j=i+2$ that shows $j>i+1$ is necessary, since the projections to $\mathcal{A}_{i+1}$ may be close. By the strong constriction property for each $\mathcal{A}_{k}$, every geodesic from $x$ to $y$ passes $\left(C+K^{\prime}\right)$-close to $\mathcal{Z}_{k}$ and $\mathcal{V}_{k}$. So every geodesic passes within $C+K^{\prime}$ of $\pi_{\mathcal{F}}(y)=\mathcal{V}_{j}$ and within $C+K^{\prime}$ of $\mathcal{Z}_{i+2}$, which is boundedly close to $\pi_{\mathcal{F}}(x)=\mathcal{V}_{i}$.

Therefore, $\pi_{\mathcal{F}}$ is $\left(1, \max \left\{d\left(\mathcal{V}_{0}, \mathcal{V}_{2}\right), C+K^{\prime}+d\left(\mathcal{V}_{0}, \mathcal{Z}_{2}\right)\right\}\right)$-strongly constricting. Lemma 3.2 says $i \mapsto f^{i}$.o is a quasigeodesic, so $f \in \Gamma$ is a strongly contracting element. 


\section{A minimal section}

Let $\mathcal{X}$ be a $G$-space with basepoint $o$. Suppose that there exists a strongly contracting element for $G \curvearrowright \mathcal{X}$. Let $\Gamma$ be an infinite normal subgroup of $G$. By Proposition 3.1, there exists a strongly contracting element $h \in \Gamma$. Let $\mathcal{H}=E(h) . o$ be an axis for $h$, and define equivariant projections to translates of $\mathcal{H}$ as in Definition 2.21. Suppose $\pi_{\mathcal{H}}$ is a $(1, C)$-strongly constricting $C$-coarse map.

Definition 4.1. For each element $g \Gamma \in G / \Gamma$ choose an element $\bar{g} \in g \Gamma$ such that $d(o, \bar{g} . o)=d(o, g \Gamma . o)=d(\Gamma . o, g \Gamma . o)$. Let $\bar{G}:=\{\bar{g} \mid g \Gamma \in G / \Gamma\}$. We call $\bar{G}$ a minimal section, and let $\overline{\mathcal{G}}$ denote $\bar{G} . o$.

Observe that $\Theta_{G / \Gamma}^{\prime}(s)=\Theta_{\bar{G}}^{\prime}(s)$, so $\delta_{G / \Gamma}=\delta_{\bar{G}}$. The next lemma says, coarsely, that the minimal section is orthogonal to translates of $\mathcal{H}$.

Lemma 4.2. For every $\bar{g} \in \bar{G}$ and for every $f \in G$ we have $d_{f \mathcal{H}}^{\pi}(o, \bar{g} . o) \leqslant 8 C+D$, where $D:=\operatorname{diam}\langle h\rangle \backslash \mathcal{H}$.

Proof. Suppose not. Then there exists an $n \neq 0$ such that

$$
D \geqslant d\left(\pi_{f \mathcal{H}}(o), f h^{n} f^{-1} . \pi_{f \mathcal{H}}(\bar{g} . o)\right) \geqslant d_{f \mathcal{H}}^{\pi}\left(o, f h^{n} f^{-1} \bar{g} . o\right)-2 C .
$$

Thus, $d_{f \mathcal{H}}^{\pi}(o, \bar{g} . o)-d_{f \mathcal{H}}^{\pi}\left(o, f h^{n} f^{-1} \bar{g} . o\right)>6 C$. However,

$$
\begin{aligned}
& d\left(o, f h^{n} f^{-1} \bar{g} . o\right) \\
& \leqslant d\left(o, \pi_{f \mathcal{H}}(o)\right)+d_{f \mathcal{H}}^{\pi}\left(o, f h^{n} f^{-1} \bar{g} . o\right)+d\left(\pi_{f \mathcal{H}}\left(f h^{n} f^{-1} \bar{g} . o\right), f h^{n} f^{-1} \bar{g} . o\right) \\
& \quad<d\left(o, \pi_{f \mathcal{H}}(o)\right)+d_{f \mathcal{H}}^{\pi}(o, \bar{g} . o)+d\left(\pi_{f \mathcal{H}}\left(f h^{n} f^{-1} \bar{g} . o\right), f h^{n} f^{-1} \bar{g} . o\right)-6 C \\
& \quad=d\left(o, \pi_{f \mathcal{H}}(o)\right)+d_{f \mathcal{H}}^{\pi}(o, \bar{g} . o)+d\left(\pi_{f \mathcal{H}}(\bar{g} . o), \bar{g} . o\right)-6 C \\
& \leqslant d(o, \bar{g} . o)
\end{aligned}
$$

This contradicts minimality of $\bar{G}$, since $f h^{n} f^{-1} \bar{g}=\bar{g} \bar{g}^{-1} f h^{n} f^{-1} \bar{g} \in \bar{g} \Gamma$.

Corollary 4.3. If $d\left(\bar{g} . o, \bar{g}^{\prime} . o\right) \geqslant 18 C+2 D$ for $\bar{g}, \bar{g}^{\prime} \in \bar{G}$, then there is no $f \in G$ such that $\bar{g} . o \in f \mathcal{H}$ and $\bar{g}^{\prime} . o \in f \mathcal{H}$.

Proof. If there were such an $f$, we would have $d_{f \mathcal{H}}^{\pi}\left(\bar{g} . o, \bar{g}^{\prime} . o\right) \geqslant 2(8 C+D)$, which means either $\bar{g}$ or $\bar{g}^{\prime}$ would contradict Lemma 4.2.

In light of Corollary 4.3, it will be convenient to pass to a coarsely dense subset of $\overline{\mathcal{G}}$ whose elements yield distinct translates of $\mathcal{H}$ :

Definition 4.4. Let $K \geqslant 18 C+2 D$, and let $A$ be a maximal subset of $\bar{G}$ such that $1 \in A$ and $d\left(\bar{g} . o, \bar{g}^{\prime} . o\right) \geqslant K$ for all distinct $\bar{g}, \bar{g}^{\prime} \in A$. Let $\mathcal{A}:=A . o$. 
By maximality, for every $\bar{g} \in \bar{G}$ there is some $a \in A$ such that $d(a . o, \bar{g} . o) \leqslant K$. There are boundedly many points of $\overline{\mathcal{G}}$ in a ball of radius $K$, so $\Theta_{\overline{\mathcal{G}}}(s)$ is bounded below by $\Theta_{\mathcal{A}}(s)$ and above by a constant multiple of $\Theta_{\mathcal{A}}(s)$. In particular, $\Theta_{\mathcal{A}}(s)$ has the same convergence behavior as $\Theta_{\overline{\mathcal{G}}}(s)$, so $\delta_{A}=\delta_{\bar{G}}=\delta_{G / \Gamma}$.

Corollary 4.3 implies $a \mathcal{H} \neq a^{\prime} \mathcal{H}$ for distinct $a, a^{\prime} \in A$.

\section{Embedding a free product set}

Let $A \subset \bar{G}$ as in Definition 4.4, and let $A^{*}:=A \backslash\{1\}$. Consider the free product set $A^{*} * \mathbb{Z}_{2}:=\bigcup_{k=1}^{\infty}\left\{\left(a_{1}, \ldots, a_{k}\right) \mid a_{i} \in A^{*}\right\}$. For any $n>0$ we can map the free product set into $G$ by $\phi_{n}:\left(a_{1}, \ldots, a_{k}\right) \mapsto a_{1} h^{n} a_{2} h^{n} \cdots a_{k} h^{n}$. Our goal is to show $\delta_{\phi_{n}\left(A^{*} * \mathbb{Z}_{2}\right)}>\delta_{A}$. We establish the inequality in the next section. In this section we show $\phi_{n}$ is an injection for all sufficiently large $n$. In fact, we prove something stronger:

Proposition 5.1. The map $A^{*} * \mathbb{Z}_{2} \rightarrow G$, sending $\left(a_{1}, \ldots, a_{k}\right) \mapsto a_{1} h^{n} \cdots a_{n} h^{n}$.o is an injection for all sufficiently large $n$.

The map is an injection because we have an action of $G$ on the quasitree $\mathcal{Y}$, and for large enough $n$ we have "quasi-edges" of the form $\left[y, y h^{n}\right]$. We have set things up so that the $a_{i}$ do not backtrack across such edges. See Figure 3. We make this precise:

Proof. Let $\underline{a}=\left(a_{1}, \ldots, a_{k}\right) \in A^{*} * \mathbb{Z}_{2}$. By Lemma 4.2 , there is a $K$ such that $d_{f \mathcal{H}}^{\pi}(o, \bar{g} . o) \leqslant K$ for every $f \in G$ and every $\bar{g} \in \bar{G}$. The choice of $A \subset \bar{G}$ in Definition 4.4 guarantees that the axes $a \mathcal{H}$ for $a \in A$ are distinct. Let $N$ be the constant of Lemma 3.6 for this $K$, and choose $n \geqslant N$.

Note that the proof of Lemma 3.6 includes the fact that

$$
d\left(o, h^{n / 2} . o\right) \geqslant K^{\prime}+K+2 C,
$$

where $K^{\prime}$ is the constant of Lemma 3.3. Therefore, if $\phi_{n}(\underline{a}) . o=\phi_{n}\left(\underline{a}^{\prime}\right) . o$, then

$$
d_{\phi_{n}(\underline{a}) \mathcal{H}}^{\pi}\left(\phi_{n}(\underline{a}) . o, \phi_{n}(\underline{a}) h^{-n / 2} . o\right)-d_{\phi_{n}(\underline{a}) \mathcal{H}}^{\pi}\left(\phi_{n}(\underline{a}) . o, \phi_{n}\left(\underline{a}^{\prime}\right) . o\right) \geq K^{\prime}+C>K^{\prime},
$$

so Lemma 3.3 implies that $\phi_{n}(\underline{a})$. $\star$ and $\phi_{n}\left(\underline{a}^{\prime}\right) . \star$, though they might not be equal, are at least contained in the same component of $\mathcal{Y} \backslash \overline{\mathcal{B}}_{\Delta}\left(\phi_{n}(\underline{a}) h^{-n / 2} . \star\right)$.

Define $\mathcal{V}_{i}(\underline{a})$ to be the $a_{1} h^{n} \cdots a_{i} h^{\infty}$ component of $\mathcal{Y} \backslash \overline{\mathcal{B}}_{\Delta}\left(a_{1} h^{n} \cdots a_{i} h^{n / 2}\right.$. $)$ for $i \leqslant k$ (recall Definition 3.5). Lemma 3.6 implies that $\mathcal{V}_{i}(\underline{a}) \supset \mathcal{V}_{i+1}(\underline{a})$ and $\phi_{n}(\underline{a}) . \star \in \mathcal{V}_{k}(\underline{a})$. Moreover, for $i \leq \min \left\{k, k^{\prime}\right\}, \mathcal{V}_{i}(\underline{a})$ and $\mathcal{V}_{i}\left(\underline{a}^{\prime}\right)$ are disjoint unless $a_{j}=a_{j}^{\prime}$ for all $j \leqslant i$.

If $\phi_{n}(\underline{a}) . o=o$, then Lemma 3.3 implies that $\star \in \mathcal{V}_{k}(\underline{a}) \subset \mathcal{V}_{1}(\underline{a})$. This contradicts the fact that $\star$ is contained in the $a_{1} h^{-\infty}$ component of $\mathcal{Y} \backslash \overline{\mathcal{B}}_{\Delta}\left(a_{1} h^{n / 2} \star \star\right)$. The same argument shows that if $\underline{a}$ is a proper prefix of $\underline{a}^{\prime}$, that is, if $\underline{a}=\left(a_{1}, \ldots, a_{k}\right)$ and $\underline{a}^{\prime}=\left(a_{1}, \ldots, a_{k}, a_{k+1}^{\prime}, \ldots, a_{k^{\prime}}^{\prime}\right)$ with $k^{\prime}>k$, then $\phi_{n}(\underline{a}) . o \neq \phi_{n}\left(\underline{a}^{\prime}\right) . o$. 


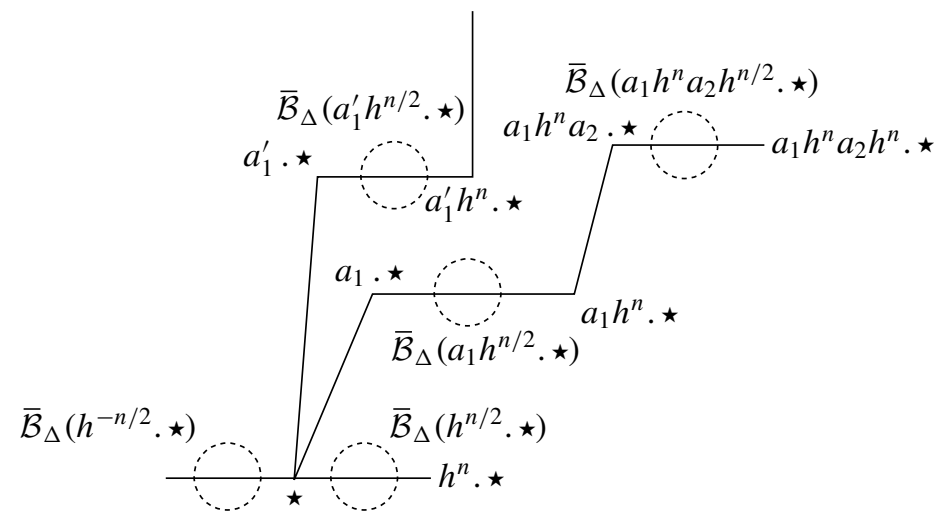

Figure 3. $A$ does not cross $h^{n}$ quasi-edges.

Suppose $\phi_{n}(\underline{a}) . o=\phi_{n}\left(\underline{a}^{\prime}\right) . o$ with $k \leqslant k^{\prime}$. Lemma 3.3 implies $\phi_{n}(\underline{a}) . \star \in \mathcal{V}_{k^{\prime}}\left(\underline{a}^{\prime}\right)$, so $a_{i}=a_{i}^{\prime}$ for all $i \leqslant k$. Since $\underline{a}$ cannot be a proper prefix of $\underline{a}^{\prime}$, we have $k=k^{\prime}$. Hence, $\phi_{n}(\underline{a}) . o=\phi_{n}\left(\underline{a}^{\prime}\right) . o$ implies $\underline{a}=\underline{a}^{\prime}$ for all sufficiently large $n$.

\section{The growth gap}

A free product of groups has greater growth exponent than the factor groups, with respect to a word metric, so we expect that $\phi_{n}\left(A^{*} * \mathbb{Z}_{2}\right)$ should have a larger growth exponent than $A$. To verify this intuition, one must show that the Poincaré series for $\phi_{n}\left(A^{*} * \mathbb{Z}_{2}\right)$ diverges at $\delta_{A}+\epsilon$ for some $\epsilon>0$. A clever manipulation of Poincaré series yields the following criterion:

Lemma 6.1 [Dal'Bo et al. 2011, Criterion 2.4; Sambusetti 2002a, Proposition 2.3]. If the map

$$
\phi_{n}: A^{*} * \mathbb{Z}_{2} \rightarrow G, \quad\left(a_{1}, \ldots, a_{k}\right) \mapsto a_{1} h^{n} \cdots a_{k} h^{n}
$$

is an injection and $\exp \left(\left|h^{n}\right| \cdot \delta_{A}\right)<\Theta_{A}\left(\delta_{A}\right)$, then $\delta_{\phi_{n}\left(A^{*} * \mathbb{Z}_{2}\right)}>\delta_{A}$.

Because our methods are coarse, we have passed to a high power $h^{n}$ of $h$ and therefore do not have control over $\left|h^{n}\right|$. However, the criterion is satisfied automatically if $A$, or, equivalently, $\bar{G}$, is divergent, which, recalling Definition 1.7, means $\Theta_{A}$ diverges at $\delta_{A}$. The following definition will be used in a condition to guarantee divergence of $\bar{G}$.

Definition 6.2. Let $\operatorname{Comp}_{Q, r}^{G} \subset G . o$ be the set of points $g . o$ such that there exists a geodesic $[x, y]$ of length $r$ with $x \in \overline{\mathcal{B}}_{Q}(o)$ and $y \in \overline{\mathcal{B}}_{Q}(g . o)$, whose interior is contained in $\mathcal{X} \backslash \overline{\mathcal{B}}_{Q}(G . o)$. Define the $Q$-complementary growth exponent of $G$ to be

$$
\delta_{G}^{c}:=\limsup _{r \rightarrow \infty} \frac{\log \# \operatorname{Comp}_{Q, r}^{G}}{r}
$$




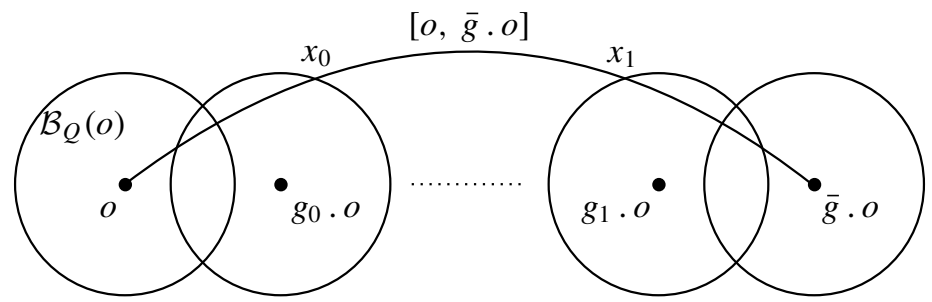

Figure 4. Splitting a geodesic into three subsegments.

Theorem 6.3. Let $G$ be a finitely generated, nonelementary group. Let $\mathcal{X}$ be a $G$-space. If $G$ contains a strongly contracting element and there exists a $Q \geqslant 0$ such that the $Q$-complementary growth exponent of $G$ is strictly less than the growth exponent of $G$, then $G \curvearrowright \mathcal{X}$ is a growth tight action.

The proof of Theorem 6.3 follows in part the proof of [Dal'Bo et al. 2011, Theorem 1.4] for geometrically finite Kleinian groups. For the divergence part of the proof, the Kleinian group ingredients of [op. cit., Theorem 1.4] are inessential, and our changes are mostly cosmetic. The real generalization is in the use of Proposition 5.1 instead of a ping-pong argument.

Proof. Let $\Gamma$ be an infinite, infinite index normal subgroup of $G$. By Proposition 3.1, there is a strongly contracting element in $\Gamma$. Let $\bar{G}$ be a minimal section of $G / \Gamma$. If $\delta_{\bar{G}} \leqslant \delta_{G}^{c}$ then we are done since $\delta_{G}^{c}<\delta_{G}$, so suppose $\delta_{\bar{G}}>\delta_{G}^{c}$.

Claim: $\bar{G}$ is divergent.

Assume the claim, and let $A$ be a maximal separated set in $\bar{G}$ as in Definition 4.4. Then $A$ and $\bar{G}$ have the same critical exponent, and are both divergent. By Proposition 5.1, for sufficiently large $n$ the map $\phi_{n}: A^{*} * \mathbb{Z}_{2} \rightarrow G$ is an injection. By Lemma 6.1, $\delta_{A}<\delta_{\phi_{n}\left(A^{*} * \mathbb{Z}\right)}$. Thus, $\delta_{G / \Gamma}=\delta_{\mathcal{A}}<\delta_{\phi_{n}\left(A^{*} * \mathbb{Z}_{2}\right)} \leqslant \delta_{G}$.

It remains to prove the claim. Let $r>0$, and suppose $d(o, \bar{g} . o)=r$. Let $0 \leqslant M_{0} \leqslant r$ and $M_{1}=r-M_{0}$. Choose a geodesic $[o, \bar{g} . o]$ from $o$ to $\bar{g} . o$, and let $[o, \bar{g} . o]\left(M_{0}\right)$ denote the point of $[o, \bar{g} . o]$ at distance $M_{0}$ from $o$.

First, we suppose that $[o, \bar{g} . o]\left(M_{0}\right) \in \mathcal{X} \backslash \overline{\mathcal{B}}_{Q}(G . o)$. Let $\left[x_{0}, x_{1}\right] \subset[o, \bar{g} . o]$ be the largest subsegment containing $[o, \bar{g} . o]\left(M_{0}\right)$ such that $\left(x_{0}, x_{1}\right) \subset \mathcal{X} \backslash \overline{\mathcal{B}}_{Q}(G . o)$. Let $m_{0}=d\left(o, x_{0}\right)$, and let $m_{1}=d\left(x_{1}, \bar{g} . o\right)$. There exist group elements $g_{i} \in G$ such that $d\left(g_{i} . o, x_{i}\right) \leqslant Q$; see Figure 4 . We have $\bar{g} . o=g_{0} \cdot g_{0}^{-1} g_{1} \cdot g_{1}^{-1} \bar{g} . o$. Now, $m_{0}-Q \leqslant d\left(o, \bar{g}_{0} . o\right) \leqslant d\left(o, g_{0} . o\right) \leqslant m_{0}+Q$, and

$$
m_{1}-Q \leqslant d\left(o, \overline{g_{1}^{-1} \bar{g}} . o\right) \leqslant d\left(o, g_{1}^{-1} \bar{g} . o\right) \leqslant m_{1}+Q .
$$

Furthermore, $g_{0}^{-1} g_{1} \in \operatorname{Comp}_{Q, r-\left(m_{0}+m_{1}\right)}^{G}$. Thus, the point $\bar{g} . o$ can be expressed as the product of an element of $\bar{G}$ of length $m_{0} \pm Q$, an element of $\bar{G}$ of length $m_{1} \pm Q$, and the quotient of an element of $\operatorname{Comp}_{Q, r-\left(m_{0}+m_{1}\right)}^{G}$. 
$(\dagger)$ The same is also true if $[o, \bar{g} . o]\left(M_{0}\right) \in \overline{\mathcal{B}}_{Q}(G . o)$, in which case we can take $m_{0}=M_{0}$ and $m_{1}=r-m_{0}$. Then choose $g_{0}=g_{1}$ so that the contribution from $\operatorname{Comp}_{Q, r-\left(m_{0}+m_{1}\right)}^{G}$ is trivial.

Let $V_{r, Q}:=\#\left(\bar{G} . o \cap \overline{\mathcal{B}}_{r+Q}(o) \backslash \mathcal{B}_{r-Q}(o)\right)$. For every $M_{0}+M_{1}=r$,

$$
V_{r, Q} \stackrel{*}{\prec} \sum_{m_{0}=0}^{M_{0}} \sum_{m_{1}=0}^{M_{1}} V_{m_{0}, Q} \cdot V_{m_{1}, Q} \cdot \# \operatorname{Comp}_{Q, r-\left(m_{0}+m_{1}\right)}^{G}
$$

Choose $\xi>0$ such that $\delta_{\bar{G}} \geqslant 2 \xi+\delta_{G}^{c}$. Since

$$
\text { \# } \operatorname{Comp}_{Q, r-\left(m_{0}+m_{1}\right)}^{G} \stackrel{*}{\prec} \exp \left(\left(r-\left(m_{0}+m_{1}\right)\right)\left(\delta_{\bar{G}}-\xi\right)\right),
$$

whenever $r-\left(m_{0}+m_{1}\right)$ is sufficiently large, it follows that

$$
\begin{aligned}
& V_{r, Q} \cdot \exp \left(-r\left(\delta_{\bar{G}}-\xi\right)\right) \stackrel{*}{\prec} \\
& \quad\left(\sum_{m_{0}=0}^{M_{0}} V_{m_{0}, Q} \cdot \exp \left(-m_{0}\left(\delta_{\bar{G}}-\xi\right)\right)\right) \cdot\left(\sum_{m_{1}=0}^{M_{1}} V_{m_{1}, Q} \cdot \exp \left(-m_{1}\left(\delta_{\bar{G}}-\xi\right)\right)\right) .
\end{aligned}
$$

Set $w_{i}:=V_{i, Q} \cdot \exp \left(-i\left(\delta_{\bar{G}}-\xi\right)\right)$ and $W_{i}:=\sum_{j=1}^{i} w_{i}$. Then, (1) and [Dal'Bo et al. 2011, Lemma 4.3] imply that $\sum_{i} w_{i} \cdot \exp (-i s)$ diverges at its critical exponent, which is

$$
\limsup _{i} \frac{\log w_{i}}{i}=\left(\limsup \frac{\log V_{i, Q}}{i}\right)-\left(\delta_{\bar{G}}-\xi\right)=\xi .
$$

So, $\infty=\sum_{i} w_{i} \cdot \exp (i \xi)=\sum_{i} V_{i, Q} \cdot \exp \left(-i \delta_{\bar{G}}\right) \stackrel{*}{`} \Theta_{\bar{G}}\left(\delta_{\bar{G}}\right)$.

Theorem 6.4. Let $G$ be a finitely generated, nonelementary group. Let $\mathcal{X}$ be a quasiconvex $G$-space. If $G$ contains a strongly contracting element then $G \curvearrowright \mathcal{X}$ is a growth tight action.

Proof. The proof is an easier special case of the proof of Theorem 6.3. If $\mathcal{X}$ is $Q$-quasiconvex, then we can always choose to be in case $(\dagger)$ of the proof.

\section{The growth of conjugacy classes}

Parkkonen and Paulin [2015] ask: given a finitely generated group $G$ with a word metric and an element $h \in G$, what is the growth rate of the conjugacy class $[h]$ of $h$ ? In a hyperbolic group $G$ there is a finite subgroup, the virtual center, consisting of elements whose centralizer is finite index in $G$. The growth exponent of a conjugacy class in the virtual center is clearly zero. Parkkonen and Paulin show that for every element $h$ not in the virtual center, $\delta_{[h]}=\frac{1}{2} \delta_{G}$. This generalizes an old result of Huber [1956] for the case of $G$ acting cocompactly on the hyperbolic plane and $h$ loxodromic. 
Since strongly contracting elements behave much like infinite order elements in hyperbolic groups, it is natural to ask whether the growth exponent of the conjugacy class of a strongly contracting element $h$ also satisfies $\delta_{[h]}=\frac{1}{2} \delta_{G}$.

We show that the lower bound holds, and the upper bound holds if $h$ moves the basepoint sufficiently far with respect to the contraction constant for the axis.

Theorem 7.1. Let $G$ be a nonelementary, finitely generated group, and let $\mathcal{X}$ be a $G$-space. Let $h$ be a strongly contracting element for $G \curvearrowright \mathcal{X}$. Then, $\delta_{[h]} \geqslant \frac{1}{2} \delta_{G}$.

Let $D:=\operatorname{diam} Z(h) \backslash \mathcal{H}$, where $Z(h)$ is the centralizer of $h$ in $G$. Suppose $\pi_{\mathcal{H}}$ is a $(1, C)$-strongly constricting, $C$-coarse map. If $d(o, h . o)>15 C+2 D$, then $\delta_{[h]}=\frac{1}{2} \delta_{G}$.

Corollary 7.2. For $h$ strongly contracting, $\delta_{\left[h^{n}\right]}=\frac{1}{2} \delta_{G}$ for all sufficiently large $n$. Proof. For $n$ nonzero, $E\left(h^{n}\right)=E(h)$ and $Z\left(h^{n}\right) \supset Z(h)$, so the same $C$ and $D$ work for $h^{n}$ as work for $h$. On the other hand, $\langle h\rangle$ is quasi-isometrically embedded, so $d\left(o, h^{n} . o\right) \asymp n$. Thus, $d\left(o, h^{n} . o\right)>15 C+2 D$ for large enough $n$.

It would be interesting to know whether the restriction on $d(o, h . o)$ is really necessary.

Question 2. Does there exist an action $G \curvearrowright \mathcal{X}$ such that $h$ is a strongly contracting element with $\delta_{[h]}>\frac{1}{2} \delta_{G}$ ?

Proof of Theorem 7.1. Define $K:=6 C+D$ and $F:=\left\{g \in G \mid d_{g \mathcal{H}}^{\pi}(o, g . o) \leqslant K\right\}$. First, we will show $\delta_{F}=\delta_{G}$. Then, we will relate $\delta_{[h]}$ to $\delta_{F}$.

For any $r \geqslant 0$ consider

$\phi:\{f \in F \backslash E(h) \mid d(o, f . o) \leqslant r\} \rightarrow\left\{g \mathcal{H} \mid g \in G \backslash E(h)\right.$ and $\left.g \mathcal{H} \cap \overline{\mathcal{B}}_{r}(o) \neq \varnothing\right\}$, defined by $\phi(f):=f \mathcal{H}$. For each axis $g \mathcal{H}$ meeting $\overline{\mathcal{B}}_{r}(o)$ there exists a $g^{\prime} \in g E(h)$ such that $d\left(o, g^{\prime} . o\right)=d(o, g \mathcal{H}) \leqslant r$. Since $\pi_{g \mathcal{H}}$ is within $5 C$ of closest point projection, by Lemma 2.8, we have $d_{g^{\prime} \mathcal{H}}^{\pi}\left(o, g^{\prime} . o\right) \leqslant 6 C \leqslant K$. Therefore, $g^{\prime} \in F$ with $\phi\left(g^{\prime}\right)=g \mathcal{H}$, so $\phi$ is surjective.

We estimate:

$$
\#\left\{\text { axes meeting } \overline{\mathcal{B}}_{r}(o)\right\} \geqslant \frac{\left.\left|G . o \cap \overline{\mathcal{B}}_{r}(o)\right| \times \# \text { axes per orbit point }\right\}}{\text { maximum number of orbit points per axis }} .
$$

The basepoint belongs to $\left[\operatorname{Stab}_{G}(o): E(h) \cap \operatorname{Stab}_{G}(o)\right]$ distinct translates of $\mathcal{H}$, so the number of axes per orbit point is constant. The maximum number of orbit points in $\overline{\mathcal{B}}_{r}(o)$ contained in a single axis is proportional to $r$, since each axis is a quasi-isometrically embedded image of a virtually cyclic group. Combined with surjectivity of $\phi$, this gives

$$
\left|F . o \cap \overline{\mathcal{B}}_{r}(o)\right| * \frac{\left|G . o \cap \overline{\mathcal{B}}_{r}(o)\right|}{r}
$$


Thus,

$$
\begin{aligned}
\delta_{F} & =\limsup _{r \rightarrow \infty} \frac{1}{r} \log \left|F . o \cap \overline{\mathcal{B}}_{r}(o)\right| \geqslant \limsup _{r \rightarrow \infty} \frac{1}{r} \log \frac{\left|G . o \cap \overline{\mathcal{B}}_{r}(o)\right|}{r} \\
& =\limsup _{r \rightarrow \infty} \frac{1}{r} \log \left|G . o \cap \overline{\mathcal{B}}_{r}(o)\right|=\delta_{G} .
\end{aligned}
$$

The reverse inequality is trivial, since $F \subset G$, so $\delta_{F}=\delta_{G}$.

Now, consider the map $\psi: F \backslash E(h) \rightarrow[h] \backslash E(h)$ defined by $\psi(f):=f h f^{-1}$. Choose minimal length representatives $e_{1}, \ldots, e_{m}$ of $Z(h) \backslash E(h)$. Associated to each $g \in G \backslash E(h)$, there is a $g^{\prime} \in g E(h)$ such that $d(o, g \mathcal{H})=d\left(o, g^{\prime} . o\right)$. There exist $z \in Z(h)$ and $i$ such that $g^{\prime}=g z e_{i}$. By setting $f:=g^{\prime} e_{i}^{-1}$, we get

$$
f h f^{-1}=g z e_{i} e_{i}^{-1} h e_{i} e_{i}^{-1} z^{-1} g^{-1}=g h g^{-1} \text {. }
$$

Since $e_{i}$ has length at most $D$ and $\pi_{g \mathcal{H}}$ is $5 C$-close to closest point projection, it follows that $f \in F$; hence, $\psi$ is surjective. Furthermore, $d\left(o, f h f^{-1} . o\right) \leqslant$ $2 d(o, f . o)+d(o, h . o)$, by the triangle inequality.

On the other hand, $\psi$ is boundedly many-to-one, since if $f h f^{-1}=f^{\prime} h f^{\prime-1}$ then $f^{\prime} \in f E(h)$, so $f \mathcal{H}=f^{\prime} \mathcal{H}$. By definition of $F$, we then have $d_{f \mathcal{H}}^{\pi}(o, f . o) \leqslant K$ and $d_{f \mathcal{H}}^{\pi}\left(o, f^{\prime} . o\right) \leqslant K$, so $d\left(f . o, f^{\prime} . o\right) \leqslant 2(C+K)$. There are uniformly boundedly many such $f^{\prime}$ for each $f$.

We conclude that $\psi$ is a surjective, boundedly-many-to-one map such that $d(o, \psi(f) . o) \stackrel{+}{\prec} 2 d(o, f . o)$ for all $f$. We excluded $E(h)$ from the domain and range, but its growth exponent is zero, since it embeds quasi-isometrically into $\mathcal{X}$, so $\delta_{[h]}=\delta_{[h] \backslash E(h)} \geqslant \frac{1}{2} \delta_{F \backslash E(h)}=\frac{1}{2} \delta_{F}=\frac{1}{2} \delta_{G}$.

Now, $d_{f \mathcal{H}}^{\pi}\left(f h . o, f h f^{-1} . o\right)=d_{f \mathcal{H}}^{\pi}(o, f . o) \leqslant K$ for $f \in F$, so

$$
d_{f \mathcal{H}}^{\pi}\left(o, f h f^{-1} . o\right)>d(f . o, f h . o)-2(C+K) .
$$

If $d(o, h . o)>15 C+2 D=C+2(C+K)$, then $d_{f \mathcal{H}}^{\pi}\left(o, f h f^{-1} . o\right)>C$, so by strong constriction,

$$
d\left(o, f h f^{-1} . o\right) \geqslant 2 d(o, f . o)+d(o, h . o)-4(C+K) .
$$

Thus, $d(o, \psi(f) . o) \succsim 2 d(o, f . o)$ and $\delta_{[h]}=\frac{1}{2} \delta_{G}$.

\section{Part II. Examples of actions with strongly contracting elements}

\section{Actions on relatively hyperbolic spaces}

Yang [2013] proved that the action of a finitely generated group $G$ with a nontrivial Floyd boundary on any of its Cayley graphs is growth tight. Relatively hyperbolic groups have nontrivial Floyd boundaries by a theorem of Gerasimov [2012], so the action of a relatively hyperbolic group on any of its Cayley graphs is growth tight. 
It is an open question whether there exists a group with a nontrivial Floyd boundary that is not relatively hyperbolic.

There is also a notion of relative hyperbolicity of metric spaces, which we will review in Section 8A. One motivating example of a relatively hyperbolic metric space is a Cayley graph of a relatively hyperbolic group. Another is the universal cover $\widetilde{M}$ of a complete, finite volume hyperbolic manifold $M$. The fundamental group $\pi_{1}(M)$ of such a manifold is a relatively hyperbolic group, so the action of $\pi_{1}(M)$ on any of its Cayley graphs is growth tight by Yang's theorem. However, this does not tell us whether the action of $\pi_{1}(M)$ on $\tilde{M}$ is growth tight. This question was addressed for a more general class of manifolds by Dal'bo, Peigné, Picaud, and Sambusetti [Dal'Bo et al. 2011], who proved growth tightness results for geometrically finite Kleinian groups. Using our main theorems, Theorem 6.3 and Theorem 6.4, we generalize their results to all groups acting on relatively hyperbolic metric spaces.

\section{A. Relatively hyperbolic metric spaces.}

Definition 8.1 (compare [Druţu 2009; Sisto 2012]). Let $\mathcal{X}$ be a geodesic metric space and let $\underline{\mathcal{P}}$ be a collection of uniformly coarsely connected subsets of $\mathcal{X}$. We say $\mathcal{X}$ is hyperbolic relative to the peripheral sets $\underline{\mathcal{P}}$ if the following conditions are satisfied:

(1) For each $A$ there exists a $B$ such that $\operatorname{diam}\left(\overline{\mathcal{B}}_{A}\left(\mathcal{P}_{0}\right) \cap \overline{\mathcal{B}}_{A}\left(\mathcal{P}_{1}\right)\right) \leqslant B$ for distinct $\mathcal{P}_{0}, \mathcal{P}_{1} \in \mathcal{P}$.

(2) There exists an $\epsilon \in\left(0, \frac{1}{2}\right)$ and $M \geqslant 0$ such that if $x_{0}, x_{1} \in \mathcal{X}$ are points such that for some $\mathcal{P} \in \underline{\mathcal{P}}$ we have $d\left(x_{i}, \mathcal{P}\right) \leqslant \epsilon \cdot d\left(x_{0}, x_{1}\right)$ for each $i$, then every geodesic from $x_{0}$ to $x_{1}$ intersects $\overline{\mathcal{B}}_{M}(\mathcal{P})$.

(3) There exist $\sigma$ and $\delta$ so that for every geodesic triangle either:

(a) there exists a ball of radius $\sigma$ intersecting all three sides, or

(b) there exists a $\mathcal{P} \in \underline{\mathcal{P}}$ such that $\overline{\mathcal{B}}_{\sigma}(\mathcal{P})$ intersects all three sides and for each corner of the triangle, the points of the outgoing geodesics from that corner which first enter $\overline{\mathcal{B}}_{\sigma}(\mathcal{P})$ are distance at most $\delta$ apart.

We say $\mathcal{X}$ is hyperbolic if it hyperbolic relative to $\underline{\mathcal{P}}=\varnothing$.

If $\mathcal{X}$ is hyperbolic in the sense of Definition 8.1, then the only nontrivial condition is (a), which is equivalent to the usual definition of hyperbolic metric space.

Definition 8.2. A group $G$ is hyperbolic relative to a collection of finitely generated peripheral subgroups if a Cayley graph of $G$ is hyperbolic relative to the cosets of the peripheral subgroups.

Sisto [2012] shows that Definition 8.2 is equivalent to Bowditch's definition [2012] of relatively hyperbolic groups. 
Definition 8.3 (compare [Groves and Manning 2007]). Let $\mathcal{X}$ be a connected graph with edges of length bounded below. A combinatorial horoball based on $X$ with parameter $a>0$ is a graph whose vertex set is Vert $\mathcal{X} \times(\{0\} \cup \mathbb{N})$, contains an edge of length 1 between $(v, n)$ and $(v, n+1)$ for all $v \in \operatorname{Vert} \mathcal{X}$ and all $n \in\{0\} \cup \mathbb{N}$, and for each edge $[v, w] \in \mathcal{X}$ contains an edge $[(v, n),(w, n)]$ of length $e^{-a n} \cdot$ length $([v, w])$.

Let $\mathcal{X}$ be hyperbolic relative to $\underline{\mathcal{P}}$. An augmented space is a space obtained from $\mathcal{X}$ as follows. By definition, there exists a constant $C$ such that each $\mathcal{P} \in \mathcal{P}$ is $C$-coarsely connected. For each $\mathcal{P} \in \underline{\mathcal{P}}$ choose a maximal subset of points that pairwise have distance at least $C$ from one another. Let these points be the vertex set of a graph. For edges, choose a geodesic connecting each pair of vertices at distance at most $2 C$ from each other. Use this graph as the base of a combinatorial horoball with parameter $a_{\mathcal{P}}>0$. The augmented space is the space obtained from the union of $\mathcal{X}$ with horoballs $\mathcal{X}_{\mathcal{P}}$ for each $\mathcal{P} \in \underline{\mathcal{P}}$ by identifying the base of $\mathcal{X}_{\mathcal{P}}$ with the graph constructed in $\mathcal{P}$.

Definition 8.4. Let $\mathcal{X}$ be a hyperbolic $G$-space, and let $\underline{P}$ be the collection of maximal parabolic subgroups of $G$. Suppose there exists a $G$-invariant collection of disjoint open horoballs centered at the points fixed by the parabolic subgroups. The truncated space is $\mathcal{X}$ minus the union of these open horoballs. We say $G \curvearrowright \mathcal{X}$ is cusp uniform if $G$ acts cocompactly on the truncated space.

If $G$ acts cocompactly on a $G$-space $\mathcal{X}^{\prime}$ that is hyperbolic relative to a $G$-invariant peripheral system $\underline{\mathcal{P}}$, then an augmented space $\mathcal{X}$ can be constructed $G$-equivariantly, and $G \curvearrowright X$ will be a cusp uniform action.

Several different versions of the following theorem occur in the literature on relatively hyperbolic groups:

Theorem 8.5 [Bowditch 2012; Groves and Manning 2008; Sisto 2012]. If $\mathcal{X}$ is hyperbolic relative to $\underline{\mathcal{P}}$, then any augmented space with horoball parameters bounded below is hyperbolic.

If $G \curvearrowright \mathcal{X}$ is a cusp uniform action, then $G$ is hyperbolic relative to the maximal parabolic subgroups and the truncated space is hyperbolic relative to boundaries of the deleted horoballs.

\section{B. Quasiconvex actions.}

Theorem 8.6. If $\mathcal{X}$ is a quasiconvex, relatively hyperbolic $G$-space and $G$ does not coarsely fix a peripheral subspace then $G \curvearrowright \mathcal{X}$ is a growth tight action.

Proof. It follows from [Sisto 2012, Lemma 5.4] that every infinite order element of $G$ that does not coarsely fix a peripheral subspace is strongly constricting. We conclude by Theorem 6.4. 
Theorem 8.6 unifies the existing proofs of growth tightness for cocompact actions on hyperbolic spaces [Sabourau 2013] and for actions of a relatively hyperbolic group on its Cayley graphs [Yang 2013], and extends to actions on a more general class of spaces.

Corollary 8.7. The action of a finitely generated group $G$ with infinitely many ends on any one of its Cayley graphs is growth tight.

Proof. Stallings' theorem [1971] says that $G$ splits nontrivially over a finite subgroup. $G$ is hyperbolic relative to the factor groups of this splitting. Since the splitting is nontrivial, $G$ does not fix any factor group, so Theorem 8.6 gives the result.

Corollary 8.7 generalizes a result of Sambusetti [2002a, Theorem 1.4], who proved it with additional constraints on the factor groups.

8C. Cusp uniform actions. Theorems 8.6 and 8.5 show that if $G \curvearrowright \mathcal{X}$ is a cusp uniform action on a hyperbolic space then the action of $G$ on the truncated space is a growth tight action. A natural question is whether $G \curvearrowright \mathcal{X}$ is a growth tight action. This action is not quasiconvex if the parabolic subgroups are infinite, as geodesics in $\mathcal{X}$ will travel deeply into horoballs, and, indeed, an example of Dal'bo, Otal, and Peigné [Dal'bo et al. 2000] shows $G \curvearrowright \mathcal{X}$ need not be growth tight.

To see how growth tightness can fail, consider the combinatorial horoball from Definition 8.3. If $\mathcal{X}$ is, say, the Cayley graph of some group and we build the combinatorial horoball with parameter $a>0$ based on $\mathcal{X}$, then the $r$-ball about a basepoint $o \in \mathcal{X}$ in the horoball metric intersected with $\mathcal{X} \times\{0\}$ contains the ball of radius $C \cdot \exp (\operatorname{ar} / 2)$ in the $\mathcal{X}$-metric, for a constant $C$ depending only on $a$. Thus, if the number of vertices of balls in $\mathcal{X}$ grows faster than polynomially in the radius, then the growth exponent with respect to the horoball metric will be infinite. Furthermore, even if growth in $\mathcal{X}$ is polynomial we can make the growth exponent in the horoball be as large as we like by taking $a$ to be sufficiently large. Dal'bo, Otal, and Peigné construct non-growth-tight examples of relatively hyperbolic groups with two cusps by taking one of the horoball parameters to be large enough so that the corresponding parabolic subgroup dominates the growth of the group; that is, the growth exponent of the parabolic subgroup is equal to the growth exponent of the whole group. Quotienting by the second parabolic subgroup then does not decrease the growth exponent, so this action is not growth tight.

Not only does this provide an example of a non-growth-tight action on a hyperbolic space, but since augmented spaces with different horoball parameters are equivariantly quasi-isometric to each other, we have:

Observation 8.8. Growth tightness is not invariant among equivariantly quasiisometric $G$-spaces.

It is shown in [Dal'Bo et al. 2011, Theorem 1.4] that this is essentially the only 
way that growth tightness can fail for cusp uniform actions. Their proof is for geometrically finite Kleinian groups, but our Theorem 6.3 generalizes this result.

Definition 8.9. Let $G \curvearrowright \mathcal{X}$ be a cusp uniform action on a hyperbolic space. Let $\underline{P}$ be the collection of maximal parabolic subgroups of $G$. Then $G \curvearrowright \mathcal{X}$ satisfies the parabolic gap condition if $\delta_{P}<\delta_{G}$ for all $P \in \underline{P}$.

Theorem 8.10. Let $G$ be a finitely generated, nonelementary group. Let $G \curvearrowright \mathcal{X}$ be a cusp uniform action on a hyperbolic space. Suppose that $G \curvearrowright \mathcal{X}$ satisfies the parabolic gap condition. Then $G \curvearrowright \mathcal{X}$ is a growth tight action.

Proof. Let $Q$ be the diameter of the quotient of the truncated space. Then the $Q$-complementary growth exponent is the maximum of the parabolic growth exponents, which, by the parabolic gap condition, is strictly less than the growth exponent of $G$. Apply Theorem 6.3.

Theorem 8.11. Let $G$ be a finitely generated group hyperbolic relative to a collection $\underline{P}$ of virtually nilpotent subgroups. Then there exists a hyperbolic $G$-space $\mathcal{X}$ such that $G \curvearrowright \mathcal{X}$ is cusp uniform and growth tight.

Proof. Construct $\mathcal{X}$ as an augmented space by taking a Cayley graph for $G$ and attaching combinatorial horoballs to the cosets of the peripheral subgroups. Since the peripheral groups are virtually nilpotent, they have polynomial growth in any word metric [Gromov 1981]. It follows that the growth exponent of each parabolic group with respect to the horoball metric is bounded by a multiple of the horoball parameter. By choosing the horoball parameters small enough, we can ensure $G \curvearrowright \mathcal{X}$ satisfies the parabolic gap condition, and apply Theorem 8.10.

8D. Beyond relative hyperbolicity. In subsequent sections we provide further examples of growth tight actions. To show these are not redundant we will verify that the groups are not relatively hyperbolic.

In this section we recall a technique for showing that a group is not relatively hyperbolic, due to Anderson, Aramayona, and Shackleton [Anderson et al. 2007]. Another approach to the question, contemporaneous to and more general than the one just cited, and also implying Theorem 8.13, was developed by Behrstock, Druțu, and Mosher [Behrstock et al. 2009].

Theorem 8.12 [Anderson et al. 2007, Theorem 2]. Let $G$ be a finitely generated, nonelementary group, and let $S$ be a (possibly infinite) generating set consisting of infinite order elements. Consider the "commutativity graph" with one vertex for each element of $S$ and an edge between vertices $s$ and $s^{\prime}$ if some nontrivial powers of $s$ and $s^{\prime}$ commute. If this graph is connected and there is at least one pair $s, s^{\prime} \in S$ such that $\left\langle s, s^{\prime}\right\rangle$ contains a rank 2 free abelian subgroup, then $G$ is not hyperbolic relative to any finite collection of proper finitely generated subgroups. 
To prove this theorem, one shows that the subgroup generated by $S$ is contained in one of the peripheral subgroups. Since $S$ generates $G$ this gives a contradiction, because the peripheral subgroups are proper subgroups of $G$.

We will actually use a mild generalization of Theorem 8.12 to the case when $S$ generates a proper subgroup of $G$ :

Theorem 8.13. Let $G$ be a finitely generated, nonelementary group. Let $S$ be a set of infinite order elements whose commutativity graph is connected and such that there is a pair $s, s^{\prime} \in S$ such that $\left\langle s, s^{\prime}\right\rangle$ contains a rank 2 free abelian subgroup. Consider the "coset graph" whose vertices are cosets of $\langle S\rangle$, with an edge connecting $g\langle S\rangle$ and $h\langle S\rangle$ if $g\langle S\rangle g^{-1} \cap h\langle S\rangle h^{-1}$ is infinite. If this graph is connected, then $G$ is not hyperbolic relative to any finite collection of proper finitely generated subgroups.

Proof. Suppose $G$ is hyperbolic relative to $\left\{P_{1}, \ldots, P_{k}\right\}$. As in the proof of Theorem 8.12, $\langle S\rangle$ is contained in a conjugate of some $P_{i}$. We assume, without loss of generality, that $\langle S\rangle \subset P_{1}$. Condition (1) of Definition 8.1 implies $P_{i} \cap g P_{i} g^{-1}$ is finite for $g \notin P_{i}$. Thus, for $g\langle S\rangle$ adjacent to $\langle S\rangle$ in the coset graph, $g \in P_{1}$ and $g\langle S\rangle g^{-1} \subset P_{1}$. Connectivity of the coset graph implies that every element of $G$ is contained in $P_{1}$, contradicting the hypothesis that $P_{1}$ is a proper subgroup.

We also note that Theorem 8.12 and Theorem 8.13 imply the, a priori, stronger result that $G$ has trivial Floyd boundary.

\section{Rank 1 actions on CAT(0) spaces}

A metric space is $C A T(0)$ if every geodesic triangle is at least as thin as a triangle in Euclidean space with the same side lengths. An isometry $\phi$ of a CAT(0) space $\mathcal{X}$ is hyperbolic if $\inf _{x \in \mathcal{X}} d(x, \phi(x))$ is positive and is attained. See, for example, [Bridson and Haefliger 1999] for more background.

Let $\mathcal{X}$ be a CAT( 0$) G$-space. Recall that our definition of " $G$-space" includes the hypothesis that $\mathcal{X}$ is proper, so an element is strongly contracting if and only if it acts as a rank 1 isometry:

Theorem 9.1 [Bestvina and Fujiwara 2009, Theorem 5.4]. Let $h$ be a hyperbolic isometry of a proper $C A T(0)$ space $\mathcal{X}$ with axis $\mathcal{A}$. Closest point projection to $\mathcal{A}$ is strongly contracting if and only if $\mathcal{A}$ does not bound an isometrically embedded half-flat in $\mathcal{X}$.

Theorems 9.1 and 6.4 show:

Theorem 9.2. If $G$ is a nonelementary, finitely generated group and $\mathcal{X}$ is a quasiconvex, $C A T(0) G$-space such that $G$ contains an element that acts as a rank 1 isometry on $\mathcal{X}$, then $G \curvearrowright \mathcal{X}$ is a growth tight action. 
Recall from Section 0C that there are many interesting classes of CAT(0) spaces that admit rank 1 isometries. In the remainder of this section we highlight a few examples.

Let $\Theta$ be a simple graph. The right angled Artin group $G(\Theta)$ defined by $\Theta$ is the group defined by the presentation

$$
\left.\left\langle g_{v} \text { for } v \in \operatorname{Vert}(\Theta)\right| g_{v} g_{w} g_{v}^{-1} g_{w}^{-1}=1 \text { for }[v, w] \in \operatorname{Edge}(\Theta)\right\rangle .
$$

The graph $\Theta$ also determines a cube complex constructed by taking a rose with one loop for each vertex of $\Theta$, and then gluing in a $k$-cube to form a $k$-torus for each complete $k$-vertex subgraph of $\Theta$. The resulting complex is called the Salvetti complex, and its fundamental group is $G(\Theta)$. The universal cover of the Salvetti complex turns out to be a CAT(0) cube complex. See [Charney 2007] for more background on right angled Artin groups.

If $\Theta$ is a single vertex then $G(\Theta) \cong \mathbb{Z}$ is elementary. If $\Theta$ is a join, that is, if it is a complete bipartite graph, then $G(\Theta)$ is a direct product of right angled Artin groups defined by the two parts. In all other cases, we find a growth tight action:

Theorem 9.3. Let $\Theta$ be a finite simple graph that is not a join and has more than one vertex. The action of the right angled Artin group $G(\Theta)$ defined by $\Theta$ on the universal cover $\mathcal{X}$ of the Salvetti complex associated to $\Theta$ is a growth tight action.

Proof. The universal cover $\mathcal{X}$ of the Salvetti complex of $\Theta$ is a cocompact, CAT( 0$)$ $G(\Theta)$-space. If $\Theta$ is not connected then $\mathcal{X}$ is hyperbolic relative to subcomplexes defined by the components of $\Theta$, so $G(\Theta) \curvearrowright \mathcal{X}$ is growth tight by Theorem 8.6. If $\Theta$ is connected then $G(\Theta)$ contains a rank 1 isometry by a theorem of Behrstock and Charney [2012]. The result follows from Theorem 9.2.

The defining graph of a right angled Artin group is a commutativity graph. If this graph is connected then the group is not relatively hyperbolic by Theorem 8.12.

A flip-graph manifold is a compact three dimensional manifold $M$ with boundary obtained from a finite collection of Seifert fibered pieces that are each a product of a circle with a compact oriented hyperbolic surface with boundary. These are glued together along boundary tori by a map exchanging the fiber and base directions. Such manifolds were studied by Kapovich and Leeb [1998], who show that the universal cover of $M$ admits a CAT( 0$)$ metric, and that an element of $\pi_{1}(M)$ that acts hyperbolically is rank 1 if and only if it is not represented by a loop contained in a single Seifert fibered piece. Thus, Theorem 9.2 implies the following:

Theorem 9.4. The action of the fundamental group of a flip-graph manifold by deck transformations on its universal cover with its natural CAT(0) metric is a growth tight action. 
To see that the fundamental group of a flip-graph manifold is not relatively hyperbolic, apply Theorem 8.13 where $S$ is the set of elliptic elements for the action of $G$ on the Bass-Serre tree of the defining graph of groups decomposition.

Theorems 9.3 and 9.4 give the first nontrivial examples of growth tight actions on spaces that are not relatively hyperbolic.

The idea of the proof for flip-graph manifolds generalizes to other CAT(0) graphs of groups via Theorem 1.14:

Theorem 9.5. Let $G$ be a nonelementary, finitely generated group that splits nontrivially as a graph of groups and is not an ascending HNN-extension. Suppose that the corresponding action of $G$ on the Bass-Serre tree of the splitting has two edges whose stabilizers have finite intersection. Suppose there exists a cocompact, CAT(0) $G$-space $\mathcal{X}$. Then, $G \curvearrowright \mathcal{X}$ is a growth tight action.

Proof. By Theorem 1.14, $G$ contains an infinite order element $h$ such that $E(h)$ is hyperbolically embedded. A theorem of Sisto [2013] implies that any axis of $h$ is a Morse quasigeodesic. An element with an axis that bounds a half-flat is not Morse, so $h$ is rank 1, and the result follows by Theorem 9.2.

\section{Mapping class groups}

Let $\mathcal{S}=\mathcal{S}_{g, p}$ be a connected and oriented surface of genus $g$ with $p$ punctures. We require $\mathcal{S}$ to have negative Euler characteristic.

Given two orientation preserving homeomorphisms $\phi, \psi: \mathcal{S} \rightarrow \mathcal{S}$, we will consider $\phi$ and $\psi$ to be equivalent if $\phi \circ \psi^{-1}$ is isotopic to the identity map on $\mathcal{S}$. Each equivalence class is called a mapping class of $\mathcal{S}$, and the set $\operatorname{Mod}(\mathcal{S})$ of all equivalence classes naturally forms a group called the mapping class group of $\mathcal{S}$.

A mapping class $f \in \operatorname{Mod}(\mathcal{S})$ is called reducible is there exists an $f$-invariant curve system on $\mathcal{S}$ and irreducible otherwise. By the Nielsen-Thurston classification of elements of $\operatorname{Mod}(\mathcal{S})$, a mapping class is irreducible and infinite order if and only if it is pseudo-Anosov [Thurston 1998].

Let $\mathcal{X}$ be the Teichmüller space of marked hyperbolic structures on $\mathcal{S}$, equipped with the Teichmüller metric. See [Hubbard 2006; Papadopoulos 2007] for more information.

Theorem 10.1 [Minsky 1996]. Each pseudo-Anosov element is strongly contracting for $\operatorname{Mod}(\mathcal{S}) \curvearrowright \mathcal{X}$.

For each $\epsilon>0$ there is a decomposition of $\mathcal{X}$ into a "thick part" $\mathcal{X} \geqslant \epsilon$ and a "thin part" $\mathcal{X}^{<\epsilon}$ according to whether the hyperbolic structure on $\mathcal{S}$ corresponding to the point $x \in \mathcal{X}$ has any closed curves of length $<\epsilon$. This decomposition is $\operatorname{Mod}(\mathcal{S})$ invariant, and $\operatorname{Mod}(\mathcal{S}) \curvearrowright \mathcal{X} \geqslant \epsilon$ is cocompact; see [Mumford 1971; Farb and Margalit 2012]. Geodesics between points in the thick part can travel deeply into the thin 
part, so the action of $\operatorname{Mod}(\mathcal{S})$ on Teichmüller space is not quasiconvex. To prove growth tightness, we need a bound on the complementary growth exponent. Such a bound is provided by Eskin, Mirzakhani, and Rafi [Eskin et al. 2012, Theorem 1.7].

Theorem 10.2. The action of the mapping class $\operatorname{group} \operatorname{Mod}(\mathcal{S})$ of $\mathcal{S}=\mathcal{S}_{g, p}$ on its Teichmüller space $\mathcal{X}$ with the Teichmüller metric is a growth tight action.

Proof. Let $\zeta=6 g-6+2 p \geqslant 2$. The growth exponent of $\operatorname{Mod}(\mathcal{S})$ with respect to its action on $\mathcal{X}$ is $\zeta$ [Athreya et al. 2012]. (We remark that the result in that reference is stated for closed surfaces, but their proof works in general. For our interest, it is enough that the growth exponent of $\operatorname{Mod}(\mathcal{S})$ is bounded below by $\zeta$. This can be obtained from [Hamenstädt 2013; Eskin et al. 2012].)

Choose $r_{0}$ and a maximal $r_{0}$-separated set in the moduli space $\operatorname{Mod}(\mathcal{S}) \backslash \mathcal{X}$, and let $\mathcal{A}$ be its full lift to $\mathcal{X}$. Given $r_{0}$ as above and $\delta=\frac{1}{2}$, let $\epsilon$ be sufficiently small, as in [Eskin et al. 2012, Theorem 1.7]. Let $Q$ be the smallest number such that the $\epsilon$-thick part of $\mathcal{X}$ is contained in $\overline{\mathcal{B}}_{Q}(\operatorname{Mod}(\mathcal{S}) . o)$. Choose a finite subset $\left\{a_{1}, \ldots, a_{n}\right\} \subset \mathcal{A}$ such that

$$
\overline{\mathcal{B}}_{Q}(o) \backslash \mathcal{B}_{Q}(\operatorname{Mod}(\mathcal{S}) . o) \subset \bigcup_{i=1}^{n} \mathcal{B}_{r_{0}}\left(a_{i}\right)
$$

Suppose that $g \in \operatorname{Mod}(\mathcal{S})$ is such that there exists a geodesic $[x, y]$ between $\overline{\mathcal{B}}_{Q}(o)$ and $\overline{\mathcal{B}}_{Q}(g . o)$ whose interior stays in $\mathcal{X} \backslash \overline{\mathcal{B}}_{Q}(\operatorname{Mod}(\mathcal{S}) . o)$. Then there are indices $i$ and $j$ such that $x \in \mathcal{B}_{r_{0}}\left(a_{i}\right)$ and $y \in \mathcal{B}_{r_{0}}\left(g . a_{j}\right)$. This means that every element contributing to $\operatorname{Comp}_{Q, r}^{\operatorname{Mod}(\mathcal{S})}$ of Definition 6.2 also contributes to some $N_{1}\left(\mathcal{Q}_{1, \epsilon}, a_{i}, a_{j}, r\right)$ of [Eskin et al. 2012, Theorem 1.7]. The conclusion of that theorem is that $N_{1}\left(\mathcal{Q}_{1, \epsilon}, a_{i}, a_{j}, r\right) \leqslant G\left(a_{i}\right) G\left(a_{j}\right) \exp \left(r \cdot\left(\zeta-\frac{1}{2}\right)\right)$ for all sufficiently large $r$, where $G$ is a particular function on $\mathcal{X}$. There are finitely many such sets, and the function $G$ is bounded on $\left\{a_{1}, \ldots, a_{n}\right\}$, so there is a constant $C$ such that $\operatorname{Comp}_{Q, r}^{\operatorname{Mod}(\mathcal{S})} \leqslant C \cdot \exp \left(r \cdot\left(\zeta-\frac{1}{2}\right)\right)$ for all sufficiently large $r$. Thus, the $Q$ complementary growth exponent is at most $\zeta-\frac{1}{2}<\zeta$. The theorem now follows from Theorems 10.1 and 6.3.

When the genus of $\mathcal{S}$ is at least 3 then there does not exist a cocompact, CAT( 0$)$ $\operatorname{Mod}(\mathcal{S})$-space [Bridson 2010]. The fact that such an $\operatorname{Mod}(\mathcal{S})$ is not relatively hyperbolic (in fact, has trivial Floyd boundary) is an application of Theorem 8.12 appearing in [Anderson et al. 2007]. Therefore, Theorem 10.2 does not follow from the results of the previous sections.

A natural question is whether the action of a mapping class group on its Cayley graphs is growth tight. There is also a combinatorial model for the mapping class group known as the marking complex. Finally, a mapping class group acts 
cocompactly on a thick part of the Teichmüller space. All of these spaces are quasiisometric, and Duchin and Rafi [2009] show that pseudo-Anosov elements are contracting for the action of a mapping class group on any one of its Cayley graphs, but we do not know whether one of these actions admits a strongly contracting element.

Question 3. Is the action of a mapping class group of a hyperbolic surface on one of its Cayley graphs/marking complex/thick part of Teichmüller space growth tight?

The outer automorphism group of a finite rank nonabelian free $\operatorname{group}, \operatorname{Out}\left(F_{n}\right)$ is often studied in analogy with $\operatorname{Mod}(\mathcal{S})$. Algom-Kfir [2011] has proven an analogue of Minsky's theorem that says that a fully irreducible outer automorphism class is strongly contracting for the action of $\operatorname{Out}\left(F_{n}\right)$ on its outer space, which is the analogue of the Teichmüller space. However, we lack the analogue of the theorem of Eskin, Mirzakhani, and Rafi that was used to control the complementary growth exponent in the mapping class group case.

There is also an analogue of the thick part of Teichmüller space called the spine of the outer space, on which $\operatorname{Out}\left(F_{n}\right)$ acts cocompactly.

Question 4. Is the action of $\operatorname{Out}\left(F_{n}\right)$ on one of its Cayley graphs/outer space/spine of outer space growth tight?

\section{Snowflake groups}

Let

$$
G:=B B(1, r)=\left\langle a, b, s, t \mid a b a^{-1} b^{-1}=1, s^{-1} a s=a^{r} b, t^{-1} a t=a^{r} b^{-1}\right\rangle
$$

be a Brady-Bridson snowflake group with $r \geqslant 3$. Let $L:=2 r$. These groups have an interesting mixture of positive and negative curvature properties. $G$ splits as an amalgam of $\mathbb{Z}^{2}=\langle a, b\rangle$ by two cyclic groups $\left\langle a^{r} b\right\rangle$ and $\left\langle a^{r} b^{-1}\right\rangle$, and the action of $G$ on the Bass-Serre tree $\mathcal{T}$ of this splitting satisfies Theorem 1.14, so $G$ has hyperbolically embedded subgroups. However, we can not automatically conclude that such a hyperbolically embedded subgroup gives rise to a strongly contracting element, as there does not exist a cocompact, $\mathrm{CAT}(0) G$-space. If such a space existed, then the Dehn function of $G$ would be at most quadratic, but Brady and Bridson [2000] have shown that the Dehn function of $B B(1, r)$ is $n^{2 \log _{2} L}>n^{2}$.

We will fix a $G$-space $\mathcal{X}$ and demonstrate two different elements of $G$ that act hyperbolically on $\mathcal{T}$ such that the pointwise stabilizer of any length 3 segment of their axes is finite. One of these elements will be strongly contracting for the action on $\mathcal{X}$, and the other will not.

Theorem 11.1. $G$ admits a cocompact growth tight action.

Observe that Theorem 8.13 with $S:=\{a, b\}$ shows that $G$ is not relatively hyperbolic. 
11A. The model space $\mathcal{X}$. Let $\mathcal{X}$ be the Cayley graph for $G$ with respect to the generating set $\left\{a, a^{r} b, a^{r} b^{-1}, s, t\right\}$, where the edges corresponding to $a^{r} b$ and $a^{r} b^{-1}$ have been rescaled to have length $L:=2 r$. The point of scaling these edges is that $a^{r} b, a^{r} b^{-1}$, and $a^{2 r}$ form an equilateral triangle of side length $L$, which will facilitate finding geodesics in this particular model.

It is also useful to consider $G$ as the fundamental group of the topological space obtained from a torus by gluing on two annuli. Choose a basepoint for the torus and for each boundary component of the annuli. For one annulus, the $s$-annulus, glue the two boundary curves to the curves $a$ and $a^{r} b$ in the torus, gluing basepoints to the basepoint of the torus. For the other annulus, the $t$-annulus, glue the two boundary curves to the curves $a$ and $a^{r} b^{-1}$ of the torus. The resulting space is a graph of spaces that Scott and Wall [1979] associated to the given graph of groups decomposition of $G$.

The fundamental group of this space is $G$, which acts freely by deck transformations on the universal cover $\mathcal{X}^{\prime}$. Choose the basepoint $o$ of $\mathcal{X}^{\prime}$ to be a lift of the basepoint of the torus. The correspondence between a vertex $g \in \mathcal{X}$ and the point $g . o \in \mathcal{X}^{\prime}$ inspires the following terminology: A plane is a coset $g\langle a, b\rangle \in G /\langle a, b\rangle$, which corresponds to a lift of the torus at the point $g . o \in \mathcal{X}^{\prime}$. An $s$-wall is the set of outgoing $s$-edges incident to a coset $g\langle a\rangle \in G /\langle a\rangle$. This corresponds to a lift of the $s$-annulus at the point $g . o \in \mathcal{X}^{\prime}$. A $t$-wall is the set of outgoing $t$-edges incident to a coset $g\langle a\rangle \in G /\langle a\rangle$. This corresponds to a lift of the $t$-annulus at the point $g . o \in \mathcal{X}^{\prime}$. Each wall separates $\mathcal{X}$ (and $\mathcal{X}^{\prime}$ ) into two complementary components. Notice that the origins of consecutive edges in an $s$-wall are connected by a single $a$-edge of length 1 , while the termini of those edges are connected by a single $a^{r} b$-edge of length $L$. We say that crossing an $s$-wall in the positive direction scales distance by a factor of $L$. The same is true for the $t$-walls.

11B. Geodesics between points in a plane. We will define a family of $\mathcal{X}$-geodesics joining 1 to every point of $\langle a, b\rangle$. This is similar to the argument of [Brady and Bridson 2000].

From the fact that $\langle a, b\rangle$ is abelian, for every point $a^{x} b^{y}$ there is a geodesic from 1 to $a^{x} b^{y}$ of the form $\left[1,\left(a^{r} b\right)^{m}\right]+\left(a^{r} b\right)^{m}\left[1,\left(a^{r} b^{-1}\right)^{n}\right]+\left(a^{r} b\right)^{m}\left(a^{r} b^{-1}\right)^{n}\left[1, a^{p}\right]$, where $[g, h]$ indicates a geodesic from $g$ to $h$.

For a point of the form $\left(a^{r} b\right)^{m}$ there is an $a^{r} b$-edge path from 1 to $\left(a^{r} b\right)^{m}$ of length $m L$. This path is clearly inefficient, as it lies along the boundary of an $s^{-1}$-wall that scales distance by $1 / L$, so we can push the original edge path across the wall to a path $s^{-1} a^{m} s$ of length $2+m$. We claim there is a geodesic from 1 to $\left(a^{r} b\right)^{m}$ of the form $\left[1, s^{-1}\right]+s^{-1}\left[1, a^{m}\right]+s^{-1} a^{m}\left[s^{-1}, 1\right]$. We have already exhibited a wall crossing path of length $2+m$, which is shorter than any path from 1 to $\left(a^{r} b\right)^{m}$ that stays in the plane $\langle a, b\rangle$. Thus, a geodesic must cross some walls. 
Every path from 1 to $\left(a^{r} b\right)^{m}$ can, by rearranging subsegments and eliminating backtracking, be replaced by a path of at most the same length and having the form $\gamma_{s}+\gamma_{t}+\gamma^{\prime}$ where:

- $\gamma_{s}=\left[1, s^{-1}\right]+s^{-1}\left[1, a^{n}\right]+s^{-1} a^{n}\left[s^{-1}, 1\right]$, if nontrivial;

- $\gamma_{t}=s^{-1} a^{n} s\left[1, t^{-1}\right]+s^{-1} a^{n} s t^{-1}\left[1, a^{p}\right]+s^{-1} a^{n} s t^{-1} a^{p}\left[t^{-1}, 1\right]$, if nontrivial;

- $\gamma^{\prime}=s^{-1} a^{n} s t^{-1} a^{p} t\left[1, a^{q}\right]$, if nontrivial.

The path $\gamma=\gamma_{s}+\gamma_{t}+\gamma^{\prime}$ is a path from 1 to

$$
s^{-1} a^{n} s t^{-1} a^{p} t a^{q}=\left(a^{r} b\right)^{n}\left(a^{r} b^{-1}\right)^{p} a^{q}=a^{r(n+p)+q} b^{n-p}=a^{r m} b^{m},
$$

so $p=n-m$ and $q=-L p$. Since $p$ and $q$ are proportional, $\gamma_{t}$ and $\gamma^{\prime}$ are either both trivial or both nontrivial. Suppose they are nontrivial. There is a symmetry that exchanges $\gamma_{t}$ with a path

$$
\gamma_{t}^{\prime}=s^{-1} a^{n} s\left[1, s^{-1}\right]+s^{-1} a^{n} s s^{-1}\left[1, a^{-p}\right]+s^{-1} a^{n} s s^{-1} a^{-p}\left[s^{-1}, 1\right]
$$

of the same length. However, $\gamma_{t}^{\prime}$ and $\gamma_{t}+\gamma^{\prime}$ have the same endpoints, and $\gamma_{t}^{\prime}$ is shorter, so $\gamma$ could not have been geodesic if $\gamma_{t}$ and $\gamma^{\prime}$ are nontrivial. Thus, if $\gamma$ is geodesic then $\gamma=\gamma_{s}$. This reduces the problem of finding a geodesic from 1 to $\left(a^{r} b\right)^{m}$ to finding a geodesic from 1 to $a^{n}$.

A similar argument holds for geodesics from 1 to $\left(a^{r} b^{-1}\right)^{m}$, so we can find geodesics from 1 to any point in $\langle a, b\rangle$ if we know geodesics from 1 to powers of $a$.

For powers of $a$ the idea is that $a^{m L},\left(a^{r} b\right)^{m}$, and $\left(a^{r} b^{-1}\right)^{m}$ form an equilateral triangle in the plane, but the latter two can be shortened by a factor of $L$ by pushing across a wall. Since $L \geq 6$, the savings of a factor of $L / 2$ in length outweighs the added overhead from crossing walls.

For small powers of $a$ we can find geodesics by inspection of the Cayley graph. For $0 \leqslant|p| \leqslant L / 2+3$, the edge path $a^{p}$ from 1 to $a^{p}$ is a geodesic of length $|p|$. For $L / 2+3 \leqslant p \leqslant L$ the edge path $s^{-1} a s t^{-1} a t a^{p-L}$ is a geodesic from 1 to $a^{p}$ of length $6+L-p$. We conclude that for $m>0$ and $-L / 2+3 \leq p \leq L / 2+3$ there is a geodesic from 1 to $a^{m L+p}$ of the form

$$
\begin{aligned}
& {\left[1, s^{-1}\right]+s^{-1}\left[1, a^{m}\right]+s^{-1} a^{m}\left[s^{-1}, 1\right]+s^{-1} a^{m} s\left[1, t^{-1}\right]} \\
& \quad+s^{-1} a^{m} s t^{-1}\left[1, a^{m}\right]+s^{-1} a^{m} s t^{-1} a^{m}\left[t^{-1}, 1\right]+s^{-1} a^{m} s t^{-1} a^{m} t\left[1, a^{p}\right]
\end{aligned}
$$

We can now find geodesics from 1 to powers of $a$ by induction, and from these we know a geodesic from 1 to any $a^{x} b^{y}$. We see an example in Figure 5, where trapezoids are walls and triangles are contained in planes. The top half boundary and bottom half boundary of the figure each give geodesics of length $5 \cdot 2^{5}-4$ between 1 and $a^{L^{5}}$. (This form of geodesic loop bears witness to the Dehn function [Brady and Bridson 2000], and inspired the name "snowflake group" [Brady et al. 2009].) 


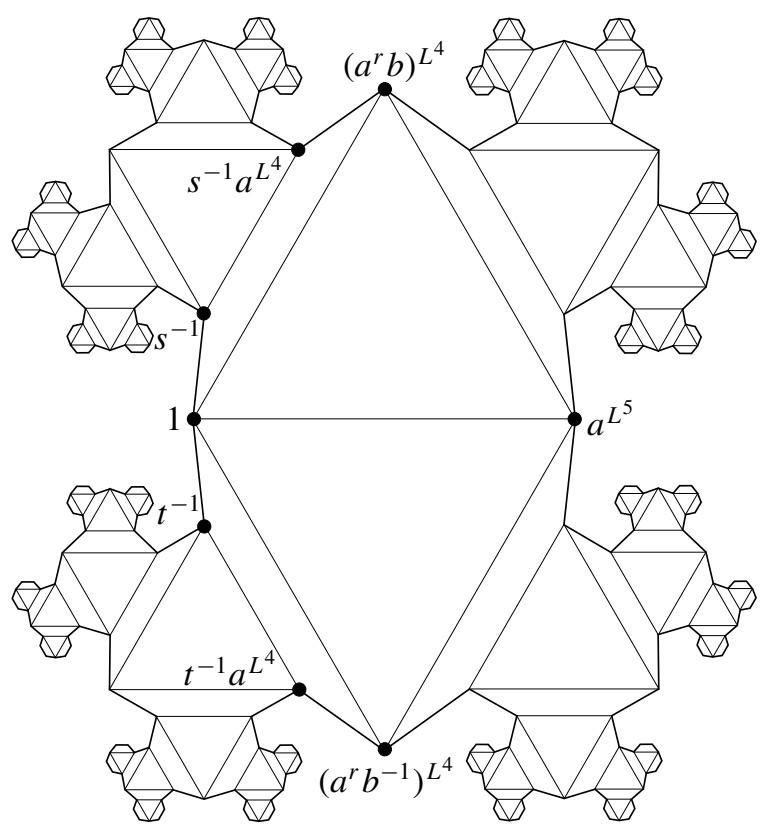

Figure 5. Snowflake: the boundary is a geodesic loop of length $2\left(5 \cdot 2^{5}-4\right)$.

11C. Projections to geodesics in $\mathcal{X}$. In this section we consider two different geodesics: $\alpha(2 n)=\left(s^{-1} t\right)^{n}$ and $\beta(n)=s^{-n}$. These are geodesics since for each of these paths, every edge crosses a distinct wall. Let $\mathcal{T}$ be the Bass-Serre tree of $G$, and let $o \in \mathcal{T}$ be the vertex fixed by the subgroup $\langle a, b\rangle$. The orbit map $g \mapsto g . o$ sends each of $\alpha$ and $\beta$ isometrically to a geodesic in $\mathcal{T}$. We will use $\pi_{\alpha}$ to denote closest point projection to $\alpha$, both in $\mathcal{X}$ and in $\mathcal{T}$, and similarly for $\beta$.

Both of these geodesics have the property that for any vertices at distance at least three in the corresponding geodesic of the Bass-Serre tree, the pointwise stabilizers of the pair of vertices is trivial. We might hope, in analogy to Theorem 9.5, that these would be strongly contracting geodesics. As in Theorem 9.5, $\left\langle s^{-1} t\right\rangle$ and $\langle s\rangle$ are hyperbolically embedded subgroups in $G$, but, of the two, we will see only $s^{-1} t$ is strongly contracting.

Geodesic $\alpha$. We claim that closest point projection $\pi_{\alpha}: \mathcal{X} \rightarrow \alpha$ is coarsely well defined and strongly contracting. First, consider $\pi_{\alpha}$ on $\langle a, b\rangle$. The geodesic $\alpha$ enters $\langle a, b\rangle$ through the incoming $t$-wall $V$ at 1 , and exits through the outgoing $s^{-1}$-wall $W$ at 1 .

Lemma 11.2. For every $v \in V$ and every $w \in W$ there exists a geodesic from $v$ to $w$ that includes the vertex 1 .

Proof. The lemma follows from the discussion of geodesics in Section 11B. 
Lemma 11.3. The orbit map $\mathcal{X} \rightarrow \mathcal{T}$ defined by $g \mapsto g$.o coarsely commutes with closest point projection to $\alpha$. In particular, closest point projection to $\alpha$ in $\mathcal{X}$ is coarsely well defined.

Proof. Suppose $z \in \mathcal{X}$ is some vertex that is separated from 1 by $V$, and suppose there is an $n \geqslant 0$ such that $\alpha(n) \in \pi_{\alpha}(z)$. Let $\sigma$ be a geodesic from $z$ to $\alpha(n)$. Write $\sigma=\sigma_{1}+\sigma_{2}+\sigma_{3}$, where $\sigma_{2}$ is the subsegment of $\sigma$ from the first time $\sigma$ crosses $V$ until the first time $\sigma$ reaches $W$. By Lemma 11.2, we can replace $\sigma_{2}$ by a geodesic segment $\sigma_{2}^{\prime}+\sigma_{2}^{\prime \prime}$ where the concatenation point is 1 . This means that $z$ is connected to $1=\alpha(0)$ by a path $\sigma_{1}+\sigma_{2}^{\prime}$. By hypothesis, the length of this path is at least the length of $\sigma$, so $\sigma_{2}^{\prime \prime}$ and $\sigma_{3}$ are trivial and $n=0$. It follows immediately that the orbit map $\mathcal{X} \rightarrow \mathcal{T}$ commutes with $\pi_{\alpha}$ up to an error of 4 . (In fact, a little more work will show the error is at most 2.)

Lemma 11.4 (bounded geodesic image property for $\pi_{\alpha}$ ). For any geodesic $\sigma$ in $\mathcal{X}$, if the diameter of $\pi_{\alpha}(\sigma . o)$ is at least 5 , then $\sigma \cap \alpha \neq \varnothing$.

Proof. Suppose $\alpha([-1,3]) . o \subset \pi_{\alpha}(\sigma . o)$. Then $\sigma$ crosses the walls $V, W, s^{-1} t V$, and $s^{-1} t W$. Write $\sigma$ as a concatenation of geodesic subsegments $\sigma_{1}+\sigma_{2}+\sigma_{3}+\sigma_{4}+\sigma_{5}$, where $\sigma_{1}$ is all of $\sigma$ prior to the first $V$ crossing, $\sigma_{2}$ is the part of $\sigma$ between the first $V$ crossing and the last $W$ crossing, $\sigma_{3}$ is the part between the last $W$ crossing and the first $s^{-1} t V$ crossing, which included edges labeled $s^{-1}$ and $t, \sigma_{4}$ is the part from the first $s^{-1} t V$ crossing until the last $s^{-1} t W$ crossing, and $\sigma_{5}$ is the remainder of $\sigma$. We can apply Section 11B to replace $\sigma_{2}$ by a geodesic $\sigma_{2}^{\prime}+\sigma_{2}^{\prime \prime}$ with the same endpoints and concatenated at 1 . Similarly, we can replace $\sigma_{4}$ by a geodesic $\sigma_{4}^{\prime}+\sigma_{4}^{\prime \prime}$ with the same endpoints and concatenated at $s^{-1} t$. But then we can replace the subsegment $\sigma_{2}+\sigma_{3}+\sigma_{4}$ of $\sigma$ by the path $\sigma_{2}^{\prime \prime}+\left[1, s^{-1} t\right]+\sigma_{4}^{\prime \prime}$ with the same endpoints. This path is strictly shorter unless $\sigma_{2}^{\prime \prime}$ and $\sigma_{4}^{\prime \prime}$ are trivial. This means that $\left[1, s^{-1} t\right] \subset \sigma \cap \alpha$.

By Proposition 2.9, this means the following:

Corollary 11.5. The element $s^{-1} t$ is strongly contracting for $G \curvearrowright \mathcal{X}$.

Together with Theorem 6.4, this proves Theorem 11.1.

Geodesic $\beta$. Using out knowledge of geodesics from Section 11B, we see that the closest point of the $s^{-1}$-wall at 1 to the point $a^{L^{k}}$ is $\left(a^{r} b\right)^{L^{k-1}}$, which is the midpoint of a geodesic from 1 to $a^{L^{k}}$. This geodesic coincides with $\beta$ on the interval from 1 to $s^{-k}$. It follows that $\pi_{\beta}\left(a^{L^{j}}\right)=\beta(j)$ for all $j \geqslant 0$.

For $0<j<k$ there is a geodesic $\sigma_{j, k}$ from $a^{L^{j}}$ to $a^{L^{k}}$ such that $d\left(\sigma_{j, k}, \beta\right)=$ $d\left(a^{L^{j}}, \beta\right)$; see Figure 6. Letting $j$ and $k-j$ grow large, the geodesics $\sigma_{j, k}$ stay outside large neighborhoods of $\beta$ but have large projections to $\beta$. Therefore, $\pi_{\beta}$ is not strongly contracting, since it does not enjoy the bounded geodesic image property. 


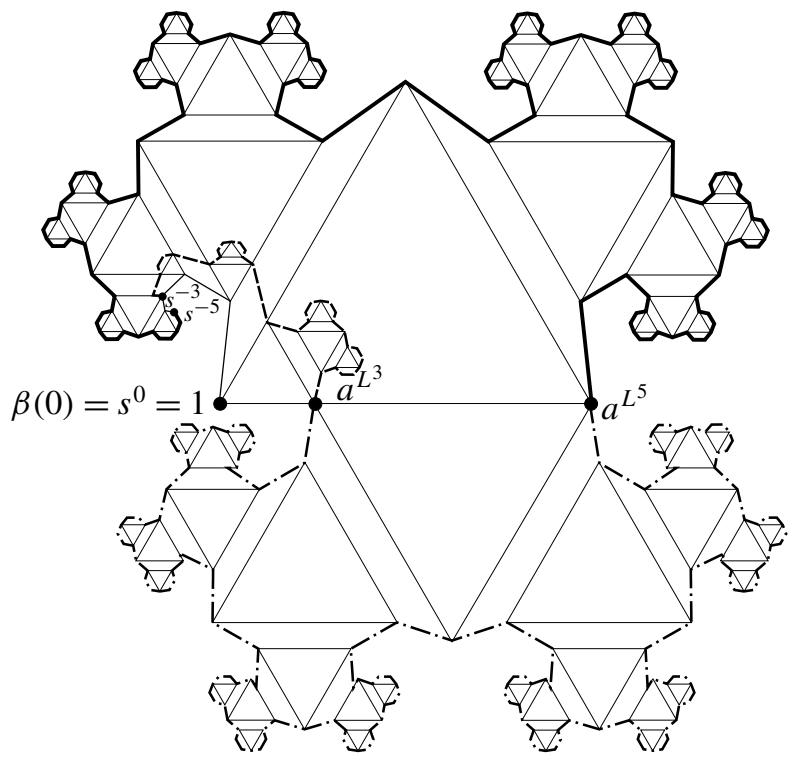

Figure 6. Geodesics $\left[a^{L^{3}}, \pi_{\beta}\left(a^{L^{3}}\right)\right]$ (dashed), $\left[a^{L^{5}}, \pi_{\beta}\left(a^{L^{5}}\right)\right]$ (solid), and $\sigma_{3,5}=\left[a^{L^{3}}, a^{L^{5}}\right]$ (dash-dot).

\section{Acknowledgments}

Arzhantseva and Cashen were supported by the European Research Council (ERC) grant of Goulnara ARZHANTSEVA, grant agreement \#259527 and the Erwin Schrödinger Institute workshop "Geometry of Computation in Groups". Cashen was also partially supported by the Austrian Science Fund (FWF):M1717-N25. Tao was supported by NSF grant DMS-1311834.

\section{References}

[Algom-Kfir 2011] Y. Algom-Kfir, "Strongly contracting geodesics in outer space", Geom. Topol. 15:4 (2011), 2181-2233. MR 2862155 Zbl 1250.20019

[Anderson et al. 2007] J. W. Anderson, J. Aramayona, and K. J. Shackleton, "An obstruction to the strong relative hyperbolicity of a group", J. Group Theory 10:6 (2007), 749-756. MR 2009c:20075 Zbl 1188.20041

[Arzhantseva and Lysenok 2002] G. N. Arzhantseva and I. G. Lysenok, "Growth tightness for word hyperbolic groups”, Math. Z. 241:3 (2002), 597-611. MR 2003h:20077 Zbl 1045.20035

[Arzhantseva and Lysenok 2006] G. N. Arzhantseva and I. G. Lysenok, "A lower bound on the growth of word hyperbolic groups", J. London Math. Soc. (2) 73:1 (2006), 109-125. MR 2006k:20088 Zbl 1093.20024

[Athreya et al. 2012] J. Athreya, A. Bufetov, A. Eskin, and M. Mirzakhani, "Lattice point asymptotics and volume growth on Teichmüller space", Duke Math. J. 161:6 (2012), 1055-1111. MR 2913101 Zbl 1246.37009 
[Ballmann 1995] W. Ballmann, Lectures on spaces of nonpositive curvature, DMV Seminar $\mathbf{2 5}$, Birkhäuser, Basel, 1995. MR 97a:53053 Zbl 0834.53003

[Ballmann and Brin 1995] W. Ballmann and M. Brin, "Orbihedra of nonpositive curvature", Inst. Hautes Études Sci. Publ. Math. 82 (1995), 169-209. MR 97i:53049 Zbl 0866.53029

[Ballmann and Buyalo 2008] W. Ballmann and S. Buyalo, "Periodic rank one geodesics in Hadamard spaces", pp. 19-27 in Geometric and probabilistic structures in dynamics, edited by K. Burns et al., Contemp. Math. 469, Amer. Math. Soc., Providence, 2008. MR 2010c:53062 Zbl 1167.53034

[Behrstock and Charney 2012] J. Behrstock and R. Charney, "Divergence and quasimorphisms of right-angled Artin groups”, Math. Ann. 352:2 (2012), 339-356. MR 2874959 Zbl 1251.20036

[Behrstock et al. 2009] J. Behrstock, C. Druţu, and L. Mosher, "Thick metric spaces, relative hyperbolicity, and quasi-isometric rigidity”, Math. Ann. 344:3 (2009), 543-595. MR 2010h:20094

[Bestvina and Fujiwara 2009] M. Bestvina and K. Fujiwara, "A characterization of higher rank symmetric spaces via bounded cohomology”, Geom. Funct. Anal. 19:1 (2009), 11-40. MR 2010m:53060 Zbl 1203.53041

[Bestvina et al. 2014] M. Bestvina, K. Bromberg, and K. Fujiwara, "Constructing group actions on quasi-trees and applications to mapping class groups", Publications mathématiques de l'IHÉS (online publication October 2014), 1-64.

[Bowditch 2012] B. H. Bowditch, "Relatively hyperbolic groups", Internat. J. Algebra Comput. 22:3 (2012), 1250016, 66. MR 2922380 Zbl 1259.20052

[Brady and Bridson 2000] N. Brady and M. R. Bridson, "There is only one gap in the isoperimetric spectrum”, Geom. Funct. Anal. 10:5 (2000), 1053-1070. MR 2001j:20046 Zbl 0971.20019

[Brady et al. 2009] N. Brady, M. R. Bridson, M. Forester, and K. Shankar, "Snowflake groups, Perron-Frobenius eigenvalues and isoperimetric spectra”, Geom. Topol. 13:1 (2009), 141-187. MR 2010a:20090 Zbl 1228.20031

[Bridson 2010] M. R. Bridson, "Semisimple actions of mapping class groups on CAT(0) spaces", pp. 1-14 in Geometry of Riemann surfaces, edited by F. P. Gardiner, London Math. Soc. Lecture Note Ser. 368, Cambridge Univ. Press, Cambridge, 2010. MR 2011i:30041 Zbl 1204.30030

[Bridson and Haefliger 1999] M. R. Bridson and A. Haefliger, Metric spaces of non-positive curvature, Grundlehren der Mathematischen Wissenschaften 319, Springer, Berlin, 1999. MR 2000k:53038 Zbl 0988.53001

[Burger and Mozes 1997] M. Burger and S. Mozes, "Finitely presented simple groups and products of trees", C. R. Acad. Sci. Paris Sér. I Math. 324:7 (1997), 747-752. MR 98g:20041 Zbl 0966.20013

[Caprace and Fujiwara 2010] P.-E. Caprace and K. Fujiwara, "Rank-one isometries of buildings and quasi-morphisms of Kac-Moody groups", Geom. Funct. Anal. 19:5 (2010), 1296-1319. MR 2011e: 20061 Zbl 1206.20046

[Caprace and Sageev 2011] P.-E. Caprace and M. Sageev, "Rank rigidity for CAT(0) cube complexes", Geom. Funct. Anal. 21:4 (2011), 851-891. MR 2012i:20049 Zbl 1266.20054

[Cashen and Tao 2014] C. H. Cashen and J. Tao, "Growth tight actions of product groups", preprint, 2014. arXiv $1404.4335 \mathrm{v} 2$

[Charney 2007] R. Charney, "An introduction to right-angled Artin groups", Geom. Dedicata 125 (2007), 141-158. MR 2008f:20076 Zbl 1152.20031

[Coulon 2013] R. Coulon, "Growth of periodic quotients of hyperbolic groups", Algebr. Geom. Topol. 13:6 (2013), 3111-3133. MR 3248727 Zbl 06213053

[Dahmani et al. 2011] F. Dahmani, V. Guirardel, and D. Osin, "Hyperbolically embedded subgroups and rotating families in groups acting on hyperbolic spaces", preprint, 2011. arXiv 1111.7048v5 
[Dal'bo et al. 2000] F. Dal'bo, J.-P. Otal, and M. Peigné, "Séries de Poincaré des groupes géométriquement finis”, Israel J. Math. 118 (2000), 109-124. MR 2001g:37040 Zbl 0968.53023

[Dal'Bo et al. 2011] F. Dal'Bo, M. Peigné, J.-C. Picaud, and A. Sambusetti, "On the growth of quotients of Kleinian groups", Ergodic Theory Dynam. Systems 31:3 (2011), 835-851. MR 2012f:37069 Zbl 1233.37021

[Druţu 2009] C. Druţu, "Relatively hyperbolic groups: geometry and quasi-isometric invariance", Comment. Math. Helv. 84:3 (2009), 503-546. MR 2011a:20111

[Duchin and Rafi 2009] M. Duchin and K. Rafi, "Divergence of geodesics in Teichmüller space and the mapping class group", Geom. Funct. Anal. 19:3 (2009), 722-742. MR 2011c:30115 Zbl 1187.30041

[Eskin et al. 2012] A. Eskin, M. Mirzakhani, and K. Rafi, "Counting closed geodesics in strata", preprint, 2012. arXiv 1206.5574v1

[Farb and Margalit 2012] B. Farb and D. Margalit, A primer on mapping class groups, Princeton Mathematical Series 49, Princeton University Press, 2012. MR 2012h:57032

[Gerasimov 2012] V. Gerasimov, "Floyd maps for relatively hyperbolic groups", Geom. Funct. Anal. 22:5 (2012), 1361-1399. MR 2989436 Zbl 1276.20050

[Grigorchuk and de la Harpe 1997] R. Grigorchuk and P. de la Harpe, "On problems related to growth, entropy, and spectrum in group theory", J. Dynam. Control Systems 3:1 (1997), 51-89. MR 98d:20039 Zbl 0949.20033

[Gromov 1981] M. Gromov, "Groups of polynomial growth and expanding maps", Inst. Hautes Études Sci. Publ. Math. 53:1 (1981), 53-73. MR 83b:53041 Zbl 0474.20018

[Groves and Manning 2007] D. Groves and J. F. Manning, "Fillings, finite generation and direct limits of relatively hyperbolic groups", Groups Geom. Dyn. 1:3 (2007), 329-342. MR 2008f:20106 Zbl 1169.20023

[Groves and Manning 2008] D. Groves and J. F. Manning, "Dehn filling in relatively hyperbolic groups”, Israel J. Math. 168 (2008), 317-429. MR 2009h:57030 Zbl 1211.20038

[Hamenstädt 2013] U. Hamenstädt, "Bowen's construction for the Teichmüller flow”, J. Mod. Dyn. 7:4 (2013), 489-526. MR 3177770 Zbl 1291.30245

[Hubbard 2006] J. H. Hubbard, Teichmüller theory and applications to geometry, topology, and dynamics, vol. 1, Matrix Editions, Ithaca, 2006. MR 2008k:30055 Zbl 1102.30001

[Huber 1956] H. Huber, "Über eine neue Klasse automorpher Funktionen und ein Gitterpunktproblem in der hyperbolischen Ebene. I", Comment. Math. Helv. 30 (1956), 20-62. MR 17,603b

[Kapovich and Leeb 1998] M. Kapovich and B. Leeb, "3-manifold groups and nonpositive curvature", Geom. Funct. Anal. 8:5 (1998), 841-852. MR 2000a:57040 Zbl 0915.57009

[Manning 2005] J. F. Manning, "Geometry of pseudocharacters", Geom. Topol. 9 (2005), 1147-1185. MR 2006j:57002 Zbl 1083.20038

[Margulis 1991] G. A. Margulis, Discrete subgroups of semisimple Lie groups, Ergebnisse der Mathematik und ihrer Grenzgebiete (3) 17, Springer, Berlin, 1991. MR 92h:22021 Zbl 0732.22008

[Minasyan and Osin 2015] A. Minasyan and D. Osin, "Acylindrical hyperbolicity of groups acting on trees", Math. Ann. 362:3-4 (2015), 1055-1105. MR 3368093

[Minsky 1996] Y. N. Minsky, "Quasi-projections in Teichmüller space", J. Reine Angew. Math. 473 (1996), 121-136. MR 97b:32020 Zbl 0848.30031

[Mumford 1971] D. Mumford, “A remark on Mahler's compactness theorem”, Proc. Amer. Math. Soc. 28 (1971), 289-294. MR 43 \#2157 Zbl 0215.23202 
[Papadopoulos 2007] A. Papadopoulos, "Introduction to Teichmüller theory, old and new", pp. 1-30 in Handbook of Teichmüller theory, vol. 1, edited by A. Papadopoulos, IRMA Lect. Math. Theor. Phys. 11, Eur. Math. Soc., Zürich, 2007. MR 2009c:30115

[Parkkonen and Paulin 2015] J. Parkkonen and F. Paulin, "On the hyperbolic orbital counting problem in conjugacy classes”, Math. Z. 279:3-4 (2015), 1175-1196. MR 3318265 Zbl 06422655

[Reinfeldt and Weidmann 2014] C. Reinfeldt and R. Weidmann, "Makanin-Razborov diagrams for hyperbolic groups", preprint, Christian-Albrechts-Universität zu Kiel, 2014, Available at http:// tinyurl.com/o7qglq9.

[Sabourau 2013] S. Sabourau, "Growth of quotients of groups acting by isometries on Gromovhyperbolic spaces”, J. Mod. Dyn. 7:2 (2013), 269-290. MR 3106714 Zbl 1286.20057

[Sambusetti 2002a] A. Sambusetti, "Growth tightness of free and amalgamated products", Ann. Sci. École Norm. Sup. (4) 35:4 (2002), 477-488. MR 2004c:20042 Zbl 1018.20036

[Sambusetti 2002b] A. Sambusetti, "Growth tightness of surface groups", Expo. Math. 20:4 (2002), 345-363. MR 2003k:57004 Zbl 1028.53033

[Sambusetti 2003] A. Sambusetti, "Growth tightness of negatively curved manifolds", C. R. Math. Acad. Sci. Paris 336:6 (2003), 487-491. MR 2004a:53048 Zbl 1030.53039

[Sambusetti 2004] A. Sambusetti, "Growth tightness in group theory and Riemannian geometry", pp. 341-352 in Recent advances in geometry and topology (Cluj-Napoca, Romania, 2003), edited by D. Andrica and P. A. Blaga, Cluj Univ. Press, 2004. MR 2005m:20078 Zbl 1086.53055

[Scott and Wall 1979] P. Scott and T. Wall, “Topological methods in group theory", pp. 137-203 in Proceedings of a Symposium on Homological and Combinational Techniques in Group Theory (Durham, 1977), edited by C. T. C. Wall, London Math. Soc. Lecture Note Ser. 36, Cambridge Univ. Press, 1979. MR 81m:57002 Zbl 0423.20023

[Sela 1999] Z. Sela, "Endomorphisms of hyperbolic groups, I: The Hopf property", Topology 38:2 (1999), 301-321. MR 99m:20081 Zbl 0929.20033

[Shukhov 1999] A. G. Shukhov, "On the dependence of the growth exponent on the length of the defining relation”, Mat. Zametki 65:4 (1999), 612-618. MR 2000i:20052

[Sisto 2011] A. Sisto, "Contracting elements and random walks", preprint, 2011. arXiv 1112.2666v2

[Sisto 2012] A. Sisto, "On metric relative hyperbolicity”, preprint, 2012. arXiv 1210.8081v1

[Sisto 2013] A. Sisto, "Quasi-convexity of hyperbolically embedded subgroups”, preprint, 2013. arXiv 1310.7753

[Stallings 1971] J. Stallings, Group theory and three-dimensional manifolds, Yale Mathematical Monographs 4, Yale University Press, New Haven-London, 1971. MR 0415622 Zbl 0241.57001

[Thurston 1998] W. P. Thurston, "Hyperbolic structures on 3-manifolds, II: Surface groups and 3-manifolds which fiber over the circle", preprint, 1998. arXiv math/9801045v1

[Yang 2013] W. Yang, "Growth tightness of groups with nontrivial Floyd boundary", preprint, 2013. arXiv $1301.5623 \mathrm{v} 1$

Received February 20, 2015.

Goulnara N. ArZhantseva

FAKULTÄT FÜR MATHEMATIK

UNIVERITÄT WIEN

OSKAR-MORGENSTERN-PLATZ 1

1090 WIEN

AUSTRIA

goulnara.arzhantseva@univie.ac.at 
Christopher H. CASHEN

FAKULTÄT FÜR MATHEMATIK

UNIVERSITÄT WIEN

OSKAR-MORGENSTERN-PLATZ 1

1090 VIENNA

AUSTRIA

christopher.cashen@univie.ac.at

JING TAO

DEPARTMENT OF MATHEMATICS

UNIVERSITY OF OKLAHOMA

601 Elm AVENUE RM 423

NORMAN, OK 73019

UNITED STATES

jing@ou.edu 


\title{
PACIFIC JOURNAL OF MATHEMATICS
}

\author{
msp.org/pjm
}

Founded in 1951 by E. F. Beckenbach (1906-1982) and F. Wolf (1904-1989)

\section{EDITORS}

Don Blasius (Managing Editor)

Department of Mathematics

University of California

Los Angeles, CA 90095-1555

blasius@math.ucla.edu

\author{
Paul Balmer \\ Department of Mathematics \\ University of California \\ Los Angeles, CA 90095-1555 \\ balmer@math.ucla.edu \\ Robert Finn \\ Department of Mathematics \\ Stanford University \\ Stanford, CA 94305-2125 \\ finn@math.stanford.edu \\ Sorin Popa \\ Department of Mathematics \\ University of California \\ Los Angeles, CA 90095-1555 \\ popa@math.ucla.edu
}

\author{
Vyjayanthi Chari \\ Department of Mathematics \\ University of California \\ Riverside, CA 92521-0135 \\ chari@math.ucr.edu \\ Kefeng Liu \\ Department of Mathematics \\ University of California \\ Los Angeles, CA 90095-1555 \\ liu@math.ucla.edu \\ Jie Qing \\ Department of Mathematics \\ University of California \\ Santa Cruz, CA 95064 \\ qing@ cats.ucsc.edu
}

\section{PRODUCTION}

Silvio Levy, Scientific Editor, production@msp.org

\section{SUPPORTING INSTITUTIONS}

ACADEMIA SINICA, TAIPEI

CALIFORNIA INST. OF TECHNOLOGY

INST. DE MATEMÁTICA PURA E APLICADA

KEIO UNIVERSITY

MATH. SCIENCES RESEARCH INSTITUTE

NEW MEXICO STATE UNIV.

OREGON STATE UNIV.

\author{
STANFORD UNIVERSITY \\ UNIV. OF BRITISH COLUMBIA \\ UNIV. OF CALIFORNIA, BERKELEY \\ UNIV. OF CALIFORNIA, DAVIS \\ UNIV. OF CALIFORNIA, LOS ANGELES \\ UNIV. OF CALIFORNIA, RIVERSIDE \\ UNIV. OF CALIFORNIA, SAN DIEGO \\ UNIV. OF CALIF., SANTA BARBARA
}

\author{
Daryl Cooper \\ Department of Mathematics \\ University of California \\ Santa Barbara, CA 93106-3080 \\ cooper@math.ucsb.edu \\ Jiang-Hua Lu \\ Department of Mathematics \\ The University of Hong Kong \\ Pokfulam Rd., Hong Kong \\ jhlu@maths.hku.hk \\ Paul Yang \\ Department of Mathematics \\ Princeton University \\ Princeton NJ 08544-1000 \\ yang@math.princeton.edu
}

These supporting institutions contribute to the cost of publication of this Journal, but they are not owners or publishers and have no responsibility for its contents or policies.

See inside back cover or msp.org/pjm for submission instructions.

The subscription price for 2015 is US \$420/year for the electronic version, and \$570/year for print and electronic.

Subscriptions, requests for back issues and changes of subscribers address should be sent to Pacific Journal of Mathematics, P.O. Box 4163, Berkeley, CA 94704-0163, U.S.A. The Pacific Journal of Mathematics is indexed by Mathematical Reviews, Zentralblatt MATH, PASCAL CNRS Index, Referativnyi Zhurnal, Current Mathematical Publications and Web of Knowledge (Science Citation Index).

The Pacific Journal of Mathematics (ISSN 0030-8730) at the University of California, c/o Department of Mathematics, 798 Evans Hall \#3840, Berkeley, CA 94720-3840, is published twelve times a year. Periodical rate postage paid at Berkeley, CA 94704, and additional mailing offices. POSTMASTER: send address changes to Pacific Journal of Mathematics, P.O. Box 4163, Berkeley, CA 94704-0163.

PJM peer review and production are managed by EditFLOW ${ }^{\circledR}$ from Mathematical Sciences Publishers.

\section{PUBLISHED BY}

\section{mathematical sciences publishers \\ nonprofit scientific publishing}

http://msp.org/

(C) 2015 Mathematical Sciences Publishers 


\section{PACIFIC JOURNAL OF MATHEMATICS}

Volume $278 \quad$ No. $1 \quad$ November 2015

Growth tight actions

Goulnara N. Arzhantseva, Christopher H. Cashen and JING TAO

A flag structure on a cusped hyperbolic 3-manifold

ELISHA FALBEL and RAFAEL SANTOS THEBALDI

A new upper bound for the Dirac operators on hypersurfaces

Nicolas GinouX, GeORGES Habib and Simon RaUlot

Games and elementary equivalence of $\mathrm{II}_{1}$-factors

ISAAC GOLDBRING and THOMAS SINCLAIR

Grossberg-Karshon twisted cubes and hesitant walk avoidance

MEGUMI HARADA and EUNJEONG LEE

Gamma factors of distinguished representations of $\mathrm{GL}_{n}(\mathbb{C})$

ALEXANDER KEMARSKY

The $W$-entropy formula for the Witten Laplacian on manifolds with 173 time dependent metrics and potentials

SONGZI LI and XIANG-DONG LI

A diagrammatic categorification of the affine $q$-Schur algebra $\hat{\boldsymbol{S}}(n, n) 201$ for $n \geq 3$

MARCO MACKAAY and ANNE-LAURE THIEL

Showing distinctness of surface links by taking 2-dimensional braids

INASA NAKAMURA

Correction to Modular $L$-values of cubic level 